Research Article

\title{
Enhanced Single-Degree-of-Freedom Analysis of Thin Elastic Plates Subjected to Blast Loading Using an Energy-Based Approach
}

\author{
M. D. Goel $\mathbb{D},{ }^{1}$ T. Thimmesh $\left(\mathbb{D},{ }^{1}\right.$ P. Shirbhate, ${ }^{1}$ and C. Bedon $\mathbb{D D}^{2}$ \\ ${ }^{1}$ Department of Applied Mechanics, Visvesvaraya National Institute of Technology (VNIT), Nagpur 440 010, India \\ ${ }^{2}$ Department of Engineering and Architecture, University of Trieste, Trieste 34127, Italy \\ Correspondence should be addressed to M. D. Goel; mdgoel@apm.vnit.ac.in
}

Received 11 May 2020; Revised 4 July 2020; Accepted 23 November 2020; Published 7 December 2020

Academic Editor: Jian Ji

Copyright (c) 2020 M. D. Goel et al. This is an open access article distributed under the Creative Commons Attribution License, which permits unrestricted use, distribution, and reproduction in any medium, provided the original work is properly cited.

\begin{abstract}
Single-degree-of-freedom (SDOF) models are known to represent a valid tool in support of design. Key assumptions of these models, on the other hand, can strongly affect the expected predictions, hence resulting in possible overconservative or misleading estimates for the response of real structural systems under extreme actions. Among others, the description of the input loads can be responsible for major design issues, thus requiring the use of more refined approaches. In this paper, a SDOF model is developed for thin elastic plates under large displacements. Based on the energy approach, careful attention is given for the derivation of the governing linear and nonlinear parameters, under different boundary conditions of technical interest. In doing so, the efforts are dedicated to the description of the incoming blast waves. In place of simplified sinusoidal pressures, the input impulsive loads are described with the support of infinite trigonometric series that are more accurate. The so-developed SDOF model is therefore validated, based on selected literature results, by analyzing the large displacement response of thin elastic plates, under several boundary conditions and real blast pressures. Major advantage for the validation of the proposed SDOF model is obtained from experimental finite element (FE) and finite difference (FD) models of literature, giving evidence of a rather good correlation and confirming the validity of the presented formulation.
\end{abstract}

\section{Introduction}

Based on continuous advances in material science and nanotechnology, modern lightweight design and engineering applications take often benefit of thin structures and load-bearing elements. Often, these structures can take the form of thin panels belonging to partitions and barriers (i.e., facades) that are subjected to severe dynamic operational conditions, such as blast loads, or extreme natural hazards. As far as these systems are characterized by a global size that is considerably large in comparison with their thickness, under blast they need to withstand high strain pressures and undergo large amplitudes of deformations. For these reasons, the response of thin plates is substantially different from classical bending and vibration formulations of linear theory [1], given that the out-of-plane deformations are no longer compatible with the small-deflection theory.
Due to the intrinsic simplicity of application of singledegree-of-freedom (SDOF) models, several formulations have been proposed in the literature for the analysis of structures under blast (see for example [2-36]). Actually, SDOF models can represent a practical tool for engineers and structural consultants. In several cases, SDOF models have been specifically developed for thin elastic plates in the nonlinear regime. Solutions have been proposed to carry out simple SDOF analysis on various structural systems under blast pressure, such as windows [2-4], composite panels [5-12], doors or walls [13-18], and so on.

With the advances in numerical analysis and computational technology, several numerical methods can be employed to obtain viable solutions (i.e., finite element (FE) or finite difference (FD) methods, etc.). However, these methods are notoriously computationally expensive and often demand high simulation expertise, both for their 
formulation and for the interpretation of results [19]. Consequently, the development of efficient and accurate SDOF models in support of first-hand investigations for thin plates (and structures in general) under large vibrations, without going into detailed numerical analysis, attracts continuous research studies. Moreover, it is worth of interest that for plates under blast loading, the Unified Facilities Criteria Design Manual [37] recommends the use of SDOF models for structural assessment purposes. The main reason for such a recommendation is that the SDOF model analysis can be developed on the basis of relatively few input parameters, in comparison with other refined but expensive calculation methods.

In the past, a number of researchers investigated the structural response of plates using various SDOF formulations. Several studies have been presented for thin plates under large amplitude vibrations (see [1, 20-34]). Different solving techniques have been generally adopted, and the response of thin elastic plates has been mostly investigated under a specific set of boundary conditions. As a further common feature of the earlier studies, the input load has been generally assumed as a sinusoidal function, thus yielding (as also shown in this paper) approximate results for blast-loaded structures.

Another key aspect is that real blast waves have a typical short duration and exponentially decaying trend, which includes both positive phase and negative (or suction) phase [10]. Blast pressures, in this regard, are commonly associated to random impulsive loads, whose description would require the use of infinite trigonometric series. Compared to simplified sinusoidal functions, the latter leads to more accurate solutions for blast-loaded structural systems in general and especially for thin structures. Accordingly, infinite trigonometric series are considered in the SDOF formulation presented herein, due the fact that it fully satisfies (differing from other approaches) a multitude of various boundary conditions. Furthermore, it is important to notice that the response of thin plates is generally more complex than other structural members. This depends on their relatively large deflections under blast and therefore the development of both membrane and bending stresses that should be properly investigated.

$\mathrm{Li}$ and Jones investigated the dynamic response of clamped circular plates subjected to blast loading, using the Johansen yield criterion which includes the effect of bending moments along with transverse shear [35]. Based on literature review [2-18, 20-35], it is important to mention that most of the available SDOF models for thin elastic plates are based on bending theory only, wherein membrane effects were considered through a bending resistance function and therefore indirectly accounted in the form of an equivalent bending stiffness only. Earlier researchers, in some other cases, employed separately the membrane effect (as a nonlinear term of the equation of motion) and explored the SDOF response of thin elastic plates under blast loading [33].

A final consideration must be spent, in conclusion, on the reliability of elastic SDOF analysis for systems under blast. Generally, structures under blast loads are primarily required to absorb part of the imposed energy in plastic regions so as to ensure acceptable and feasible design performances. Therefore, a detailed damage analysis is often required. On the other hand, the same issue also requires first-hand elastic analysis, which is the starting point of more complex elastic-plastic studies. In some cases (i.e., when brittle elastic materials are used, like for example glass), elastic analysis represents the reference calculation approach in support of design, to avoid fracture.

In this paper, past literature efforts are further extended, and an enhanced SDOF model is developed based on energy principles, by employing infinite trigonometric series for the blast loading description. The proposed method is validated towards analytical, experimental, and numerical data of literature, for both static calculations (i.e., stiffness parameters) and dynamic loading configurations (i.e., field blast experiments of literature). The enhanced SDOF model is assessed for SS (simply supported) or CC (clamped) plates, with respect to available literature results. As shown, a rather good agreement is generally found for all of them, thus conforming the accuracy of the proposed method. Moreover, with suitable modification, the same SDOF procedure can be further adapted to predict the response of thin elastic plates under pulse loading, thus further enforcing the potential of the approach.

\section{Problem Definition}

It is well known that a SDOF system requires the definition of only one coordinate to describe its position at any instant of time, $t$. Moreover, if the basic components of the vibratory system behave linearly (i.e., spring stiffness, mass, and damping (if any)), the resulting behavior is known to be linear (Figure 1(a)). In this case, the differential equation that governs the mechanical response can be solved by means of the principle of superposition.

However, if any of the basic components behave nonlinearly, the overall behavior of the SDOF system is obviously nonlinear, and appropriate calculation tools are required (Figure 1(b)). Further, while considering the bending stiffness only, the system is still known as a linear system, wherein the system along with membrane effect is an example of a nonlinear system in this study.

The present paper focuses on thin rectangular, isotropic elastic plates with dimensions $a \times b$ and thickness $h$ (Figure 2(a)), under various boundary conditions (Figure 2(b)). The input blast wave is described in the form of a distributed, time-varying pressure $q(x, y, t)$, with $x, y$ being the Cartesian coordinates and $t$ being the time parameter.

The nonlinear elastic dynamic behavior of a representative plate (see Figure 2(a)) can be efficiently expressed by von Karman's equations:

$$
\begin{aligned}
& \rho h\left(\frac{\partial^{2} w}{\partial t^{2}}\right)+\left(\frac{E h^{3}}{12\left(1-v^{2}\right)}\right) \nabla^{4} w-h\left(\frac{\partial}{\partial x_{\beta}}\right)\left(\sigma_{\alpha \beta}\left(\frac{\partial w}{\partial x_{\alpha}}\right)\right) \\
& -q(x, y, t)=0,
\end{aligned}
$$




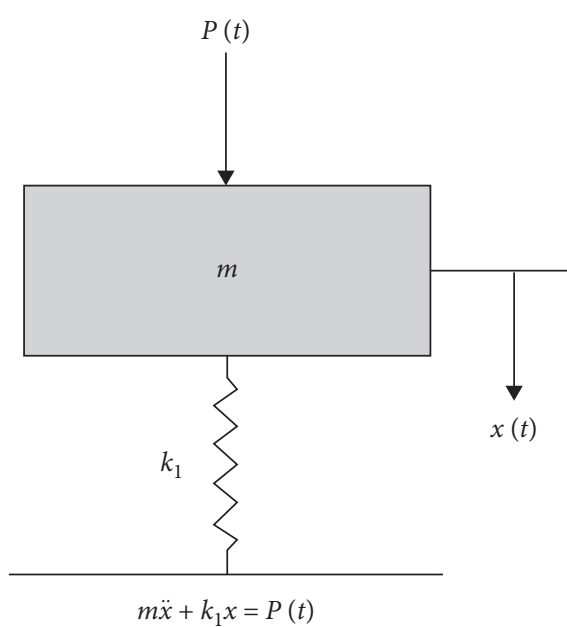

(a)

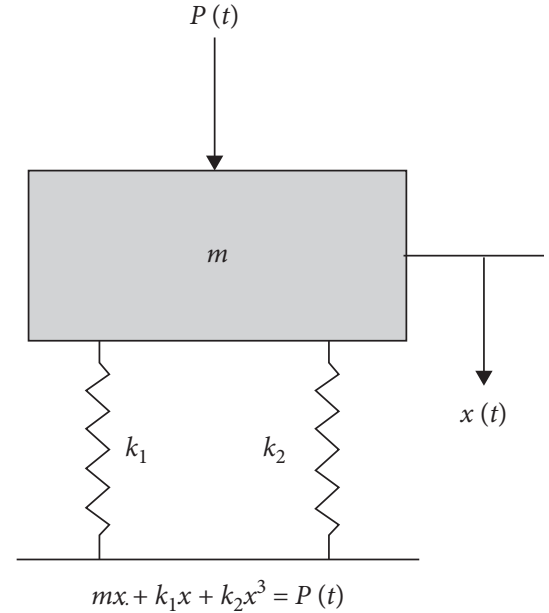

(b)

Figure 1: Governing differential equations for (a) linear and (b) nonlinear SDOF models.

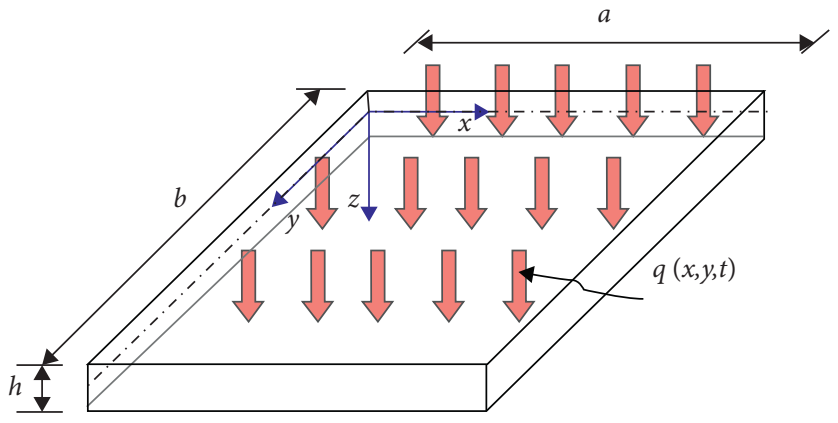

(a)

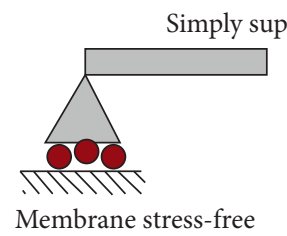

Membrane stress-free

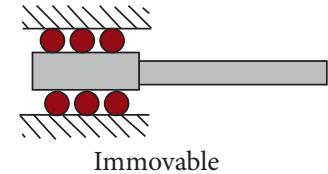

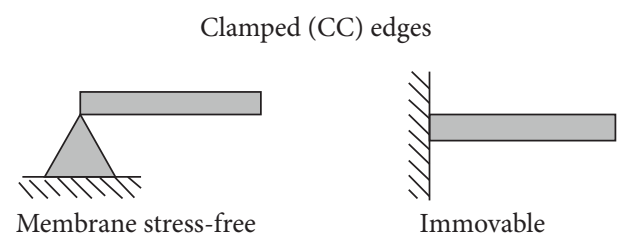

Membrane stress-free

(b)

Figure 2: (a) Reference thin elastic plate under uniform pressure (q), with evidence of (b) the selected edge boundary conditions.

$$
\left(\frac{\partial \sigma_{\alpha \beta}}{\partial x_{\beta}}\right)=0
$$

where $E$ is Young's modulus, $\nu$ is Poisson's ratio, and $\rho$ is the mass density for the material in use. Moreover, $w$ is the outof-plane deflection (middle plane of the plate) under the external normal force per unit area $q$, while $\sigma_{\alpha \beta}$ is Cauchy's stress tensor (with $\alpha, \beta$ being the subscripts that take the value of 1 or 2 ). The bending stiffness $D$ of the plate is given by

$$
D=\left(\frac{E h^{3}}{12\left(1-v^{2}\right)}\right) \text {. }
$$

Finally, the two-dimensional biharmonic operator in equation (1) is defined as

$$
\nabla^{4} w=\left(\frac{\partial^{4} w}{\partial x^{4}}\right)+2\left(\frac{\partial^{4} w}{\partial x^{2} \partial y^{2}}\right)+\left(\frac{\partial^{4} w}{\partial y^{4}}\right)
$$

Equation (4) can be derived from kinematic assumptions and constitutive relations for the plate and assumes that the out-of-plane stresses $\left(\sigma_{33}, \sigma_{13}\right.$, and $\left.\sigma_{23}\right)$ are zero. Together with equations (1) and (2), it is able to express the conservation of linear momentum in two dimensions. Further, the three Föppl-von Karman equations $[1,21,26]$ can be reduced to two, by introducing Airy's stress function $F(x, y, t)$, as defined by 


$$
\begin{aligned}
& \sigma_{x x}=\left(\frac{\partial^{2} F}{\partial x^{2}}\right), \\
& \sigma_{x y}=\left(\frac{\partial^{2} F}{\partial x \partial y}\right), \\
& \sigma_{y y}=\left(\frac{\partial^{2} \varphi}{\partial y^{2}}\right),
\end{aligned}
$$

where $\sigma_{x x}, \sigma_{x y}$, and $\sigma_{y y}$ are membrane stresses induced in the plate. Accordingly, the above equations can be rewritten as

$$
\begin{aligned}
D(w, F) & =\frac{E h^{3}}{12\left(1-v^{2}\right)} \nabla^{2} w-h\left(\frac{\partial^{2} F}{\partial y^{2}}\right)\left(\frac{\partial^{2} w}{\partial x^{2}}+\frac{\partial^{2} F}{\partial x^{2}} \frac{\partial^{2} w}{\partial y^{2}}\right)-2 \frac{\partial^{2} F}{\partial x \partial y}\left(\frac{\partial^{2} w}{\partial x \partial y}\right), \\
-q(x, y, t) & =0, \\
\nabla^{2} F+E\left\{\left(\frac{\partial^{2} w}{\partial x^{2}}\right)\left(\frac{\partial^{2} w}{\partial y^{2}}\right)-\left(\frac{\partial^{2} w}{\partial x \partial y}\right)^{2}\right\} & =0 .
\end{aligned}
$$

Further, the corresponding membrane forces in the plate can be expressed as

$$
\begin{aligned}
& N_{11}=h\left(\frac{\partial^{2} F}{\partial y^{2}}\right), \\
& N_{12}=-h\left(\frac{\partial^{2} F}{\partial x \partial y}\right), \\
& N_{22}=h\left(\frac{\partial^{2} F}{\partial x^{2}}\right),
\end{aligned}
$$

where Airy's stress function represents the membrane action in the plate that is induced by large displacements only.

As also schematized in Figure 2(b), the boundary conditions of technical interest considered in the present investigation are defined in equations (8)-(20). For all the examined edge restraints, the coordinate system in Figure 2(a) is taken into account.

For out-of-plane displacement of the plate, the following boundary configurations are defined (see also Figure 2(b)):

(i) Simply supported (SS) edge condition: all the edges are linearly simply supported.

$$
\begin{array}{r}
\left.\mathbf{w}(\mathbf{x}, \mathbf{y}, \mathbf{t})\right|_{\mathbf{x}=0} ^{\mathrm{x}=\mathbf{a}}=0 \\
{\left.\left[\left(\frac{\partial^{2} \mathbf{w}(\mathbf{x}, \mathbf{y}, \mathbf{t})}{\partial \mathbf{x}^{2}}\right)+v\left(\frac{\partial^{2} \mathbf{w}(\mathbf{x}, \mathbf{y}, \mathbf{t})}{\partial \mathbf{y}^{2}}\right)\right]\right|_{x=0} ^{\mathbf{x}=\mathbf{a}}=0}
\end{array}
$$

$$
\begin{array}{r}
\left.\mathbf{w}(\mathbf{x}, \mathbf{y}, \mathbf{t})\right|_{\mathbf{y}=0} ^{\mathbf{y}=\mathbf{b}}=0, \\
{\left.\left[\frac{\partial^{2} \mathbf{w}(\mathbf{x}, \mathbf{y}, \mathbf{t})}{\partial \mathbf{y}^{2}}+v \frac{\partial^{2} \mathbf{w}(\mathbf{x}, \mathbf{y}, \mathbf{t})}{\partial \mathbf{x}^{2}}\right]\right|_{\mathbf{y}=0} ^{\mathbf{y}=\mathbf{b}}=0 .}
\end{array}
$$

(ii) Clamped (CC) edge condition: all the edges are linearly clamped.

$$
\begin{aligned}
& \left.\mathbf{w}(\mathbf{x}, \mathbf{y}, \mathbf{t})\right|_{\mathbf{x}=0} ^{\mathbf{x}=\mathbf{a}}=0,\left.\quad\left[\frac{\partial \mathbf{w}(\mathbf{x}, \mathbf{y}, \mathbf{t})}{\partial \mathbf{x}}\right]\right|_{\mathbf{x}=0} ^{\mathbf{x}=\mathbf{a}}=0, \\
& \left.\mathbf{w}(\mathbf{x}, \mathbf{y}, \mathbf{t})\right|_{\mathbf{y}=0} ^{\mathbf{y}=\mathbf{b}}=0,\left.\quad\left[\frac{\partial \mathbf{w}(\mathbf{x}, \mathbf{y}, \mathbf{t})}{\partial \mathbf{y}}\right]\right|_{\mathbf{y}=0} ^{\mathbf{y}=\mathbf{b}}=0 .
\end{aligned}
$$

As far as the membrane stresses are also taken into account, the reference boundary conditions are defined as follows (see also Figure 2(b)):

(iii) Immovable edge condition: all the edges are assumed to be immovable, according to equations (12) and (13). It is important to notice that this edge condition incorporates membrane stresses.

$$
\begin{aligned}
& \left.u(x, y, t)\right|_{x=0} ^{x=a}=0,\left.\quad\left[\sigma_{m}^{x y}=\frac{\partial^{2} F}{\partial x \partial y}\right]\right|_{x=0} ^{x=a}=0, \\
& \left.v(x, y, t)\right|_{y=0} ^{y=b}=0,\left.\quad\left[\sigma_{m}^{x y}=\frac{\partial^{2} F}{\partial x \partial y}\right]\right|_{y=0} ^{y=b}=0 .
\end{aligned}
$$

(iv) Membrane stress-free edge condition: all the edges are assumed free from membrane stresses, and this condition is defined by equations (14) and (15). 


$$
\begin{gathered}
\sigma_{x x}^{m}=\left.\frac{\partial^{2} F}{\partial y^{2}}\right|_{x=0} ^{x=a}=0,\left.\quad\left[\sigma_{x y}^{m}=\frac{\partial^{2} F}{\partial x \partial y}\right]\right|_{x=0} ^{x=a}=0, \\
\sigma_{y y}^{m}=\left.\frac{\partial^{2} F}{\partial x^{2}}\right|_{y=0} ^{y=b}=0,\left.\quad\left[\sigma_{x y}^{m}=\frac{\partial^{2} F}{\partial x \partial y}\right]\right|_{y=0} ^{y=b}=0 .
\end{gathered}
$$

(v) Movable edge condition: all the edges are assumed movable, as defined by equations (16)-(19). Further, it is to be noted that the movable edges are the edges which are kept straight by a distribution of normal stresses, whose resultant is zero.

$$
\begin{gathered}
\left.u(x, y, t)\right|_{x=0} ^{x=a} \neq 0 \text { (Constant), }\left.\quad\left[\sigma_{m}^{x y}=\frac{\partial^{2} F}{\partial x \partial y}\right]\right|_{x=0} ^{x=a}=0, \\
\left.v(x, y, t)\right|_{y=0} ^{y=b} \neq 0 \text { (Constant), }\left.\quad\left[\sigma_{m}^{x y}=\frac{\partial^{2} F}{\partial x \partial y}\right]\right|_{y=0} ^{y=b}=0, \\
P_{x}(x, t)=\left.h \int_{0}^{b} \frac{\partial^{2} F}{\partial y^{2}} \mathrm{~d} y\right|_{x=0} ^{x=a}=0, \\
P_{y}(y, t)=\left.h \int_{0}^{a} \frac{\partial^{2} F}{\partial x^{2}} \mathrm{~d} y\right|_{y=0} ^{y=b}=0,
\end{gathered}
$$

where $P_{x}$ and $P_{y}$ are the resultant forces in the $x$ and $y$ directions, respectively.

Further, at the time $t=0$ (i.e., the time instant at which the plate is at rest), the out-of-plane deflections, $w$ of the middle plane, of the plate are defined as

$$
\begin{aligned}
\left.\mathbf{w}(\mathbf{x}, \mathbf{y}, \mathbf{t})\right|_{\mathbf{t}=0} & =0, \\
{\left.\left[\frac{\partial \mathbf{w}(\mathbf{x}, \mathbf{y}, \mathbf{t})}{\partial \mathbf{t}}\right]\right|_{\mathbf{t}=0} } & =0 .
\end{aligned}
$$

Therefore, the investigation of thin elastic plates under large deflections can be generally solved using two nonlinear differential equations (i.e., equations (1) and (2)) with various edge boundaries and membrane stress configurations of technical interest (i.e., equations (8)-(19)), along with their initial condition (as given by equation (20)).

\section{Blast Load Description}

Among the available SDOF formulations for thin elastic plates under impulsive loads, various formulations and assumptions can be found in the literature. Besides, it is generally recognized that the analytical solution of the complex governing equations, as well as their numerical solution, involves considerable computational difficulties.

Within the multitude of existing SDOF formulations, a critical examination highlights that most of them are developed on the basis of a sinusoidal load function (see, for example, [25-27, 33]). Minimum variations can be found in the reference Cartesian system. As far as the reference $x, y$ axes are located at the central point of the midplane of the plate in Figure 2(a), for example, the outof-plane deflections, $w$, are commonly expressed as $[25-27,33]$

$$
w(x, y, t)=w_{0}(t) \int_{0}^{a} \int_{0}^{b} \sin \left(\frac{\pi x}{a}\right) \sin \left(\frac{\pi y}{b}\right) \mathrm{d} x \mathrm{~d} y .
$$

Conversely, when the external load is described considering reference coordinate system from Bayles et al. [26], the resulting displacement of the plate is given by (i.e., $[1,33])$

$$
w(x, y, t)=w_{0}(t) \int_{-(a / 2)}^{(a / 2)} \int_{-(b / 2)}^{(b / 2)} \cos \left(\frac{\pi x}{a}\right) \cos \left(\frac{\pi y}{b}\right) \mathrm{d} x \mathrm{~d} y .
$$

As such, all the above SDOF formulations are equivalent. Moreover, they are able to provide solutions for plates under sinusoidal loads only.

The latter assumption represents one of the major issues for most of the SDOF formulations of literature, given that the assumed functions, even for sinusoidal loads only, typically result in rather approximate predictions. The reason for this weakness (as also discussed in this paper) derives from the lack of convergence of finite trigonometric series that take the form of a sinusoidal function when $m=1$ and $n=1$. Otherwise, the convergence is always ensured by infinite trigonometric series. Such a concept is also in contrast with blast-related issues, given that blast pressures are recognized to act as random impulsive loads, thus recommending the use of infinite trigonometric series for enhanced solutions. 
Accordingly, the present research study focuses on the derivation of linear and nonlinear governing parameters for SDOF systems subjected to blast loading, based on energy concepts and infinite trigonometric series.

To this aim, the adopted functions for SS and CC plates are expressed by equations (23) and (24), respectively.

$$
\begin{aligned}
& \varphi(x, y)=\sum_{m=1}^{\infty} \sum_{n=1}^{\infty} \sin \left(\frac{m \pi x}{a}\right) \sin \left(\frac{n \pi y}{b}\right), \\
& \varphi(x, y)=\sum_{m=1}^{\infty} \sum_{n=1}^{\infty} \sin ^{2}\left(\frac{m \pi x}{a}\right) \sin ^{2}\left(\frac{n \pi y}{b}\right) .
\end{aligned}
$$

Using this function, the blast response of thin elastic plates is expected to vary in time, but the governing parameters will remain constant. Moreover, the advantage of equations (23) and (24) is that the chosen function is able to satisfy all the boundary conditions in Figure 2(b), for both SS and CC plates (for any value of $m$ and $n$ ). Further, the components of the three-dimensional Green-Lagrangian strain tensor are defined similar to [33].

\section{Nonlinear SDOF Model}

In the present investigation, it is assumed that the out-ofplane, transverse deflection, $w$, for the plate in Figure 2(a) can be described as

$$
w(x, y, t)=Y(t) \varphi(x, y)
$$

where $Y(t)$ is a function of time only, which needs to be determined, and $\varphi(x, y)$ is the deflection shape function, as defined by equations (23) and (24) for the SS and CC conditions, respectively. The deflection shape functions are assumed in terms of sine function (whereas, Feldgun et al. assumed in [33] a cosine function). As such, the following ordinary nonlinear differential equation is obtained to describe the behavior of the reference SDOF:

$$
m \ddot{Y}(t)+K_{1} Y(t)+K_{3} Y^{3}(t)=P(t) .
$$

4.1. Simply Supported (SS) Plate. Assume a deflection shape function for a thin elastic rectangular plate under blast loading as per equation (23). The mass $m$ is therefore defined as

$$
m=\left(\frac{\rho a b h}{a b}\right) \int_{0}^{a} \int_{0}^{b} \varphi^{2}(x, y) \mathrm{d} x \mathrm{~d} y
$$

or

$$
\begin{aligned}
m= & \left(\frac{\rho a b h}{4 a b}\right) \int_{0}^{a} \int_{0}^{b} \sum_{m=1}^{\infty} \sum_{n=1}^{\infty}\left(1-\cos \left(\frac{2 m \pi x}{a}\right)\right. \\
& -\cos \left(\frac{2 n \pi y}{b}\right)+\cos \left(\frac{2 m \pi x}{a}\right) \cos \left(\frac{2 n \pi y}{b}\right) \mathrm{d} x \mathrm{~d} y .
\end{aligned}
$$

Integrating equation (28), it is found that

$$
\left.\left.m=\left(\frac{\rho a b h}{4 a b}\right) \sum_{m=1}^{\infty} \sum_{n=1}^{\infty} x y-y\left(\frac{\sin (2 m \pi x / a)}{(2 m \pi / a)}\right)-x\left(\frac{\sin (2 m \pi y / b)}{(2 m \pi / b)}\right)+\frac{\sin (2 m \pi x / a) \sin (2 m \pi y / b)}{(2 m \pi / a)(2 m \pi / b)}\right]_{0}^{a}\right]_{0}^{b} .
$$

By applying the above limits, equation (29) reduces to

$$
m=\left(\frac{\rho a b h}{4}\right)
$$

Therefore, equation (30) expresses the mass of a thin, rectangular, and elastic isotropic plate with dimensions $a \times b$, thickness $h$, and density $\rho$ under a given SS edge condition. Further, a given external normal force per unit area of the plate, $P$, can be expressed as

$$
P(t)=\int_{0}^{a} \int_{0}^{b} q(x, y, t) \varphi(x, y) \mathrm{d} x \mathrm{~d} y .
$$

In this study, it is assumed that the distributed pressure $q(x, y, t)$ may be described as

$$
q(x, y, t)=Q(x, y) P(t),
$$

where in case of uniform lateral pressure, it is $Q(x, y)=1$, and therefore:

$$
P(t)=\int_{0}^{a} \int_{0}^{b} \sum_{m=1}^{\infty} \sum_{n=1}^{\infty} \sin \left(\frac{m \pi x}{a}\right) \sin \left(\frac{n \pi y}{b}\right) \mathrm{d} x \mathrm{~d} y P_{1}(t) \mathrm{d} t,
$$

where $P_{1}(t)$ represents an exponentially decaying real blast function agreeing with [10]. Integrating the above equation and applying the reference limits, it is therefore found that

$$
P(t)=\left(\frac{4 a b}{(\pi)^{2}}\right) \sum_{m=1,3,5, \ldots} \sum_{n=1,3,5, \ldots}\left(\frac{1}{m n}\right) P_{1}(t) \mathrm{d} t,
$$

where equation (34) represents the external normal force per unit area of the plate in trigonometric series.

Accordingly, the plate bending stiffness $K_{1}$ can be expressed as 


$$
K_{1}=D \int_{0}^{a} \int_{0}^{b}\left\{\left[\left(\frac{\partial^{2} \varphi(x, y)}{\partial x^{2}}\right)+\left(\frac{\partial^{2} \varphi(x, y)}{\partial y^{2}}\right)\right]^{2}-2(1-\nu)\left[\begin{array}{c}
\left(\frac{\partial^{2} \varphi(x, y)}{\partial x^{2}}\right)\left(\frac{\partial^{2} \varphi(x, y)}{\partial y^{2}}\right) \\
-\left[\frac{\partial^{2} \varphi(x, y)}{\partial x \partial y}\right]^{2}
\end{array}\right]\right\} \mathrm{d} x \mathrm{~d} y .
$$

Applying the assumed deflection function (equation (23)) and solving equation (34), $K_{1}$ for SS plates is finally expressed as

$$
K_{1}=\left(\frac{\pi^{4} D a b}{4}\right)\left\{\sum_{m=1}^{\infty} \sum_{n=1}^{\infty}\left[\left(\frac{m}{a}\right)^{2}+\left(\frac{n}{b}\right)^{2}\right]^{2}\right\} .
$$

In order to obtain the membrane stiffness function, a suitable stress function for large deflections of SS plates needs first to be considered. The substitution of deflection (i.e., equations (23) and (25) for the SS condition) into equation (2) yields

$$
\begin{aligned}
& {\left[\left(\frac{\partial^{4} F}{\partial x^{4}}\right)+2\left(\frac{\partial^{4} F}{\partial x^{2} \partial y^{2}}\right)+\left(\frac{\partial^{4} F}{\partial y^{4}}\right)\right]} \\
& =E\left[\left\{\frac{\partial^{2} w}{\partial x \partial y}\right\}^{2}-\left(\frac{\partial^{2} w}{\partial x^{2}}\right)\left(\frac{\partial^{2} w}{\partial y^{2}}\right)\right] .
\end{aligned}
$$

Herein:

$$
\begin{aligned}
\left(\frac{\partial^{2} w(x, y)}{\partial x^{2}}\right) & =-\sum_{m=1}^{\infty} \sum_{n=1}^{\infty}\left(\frac{m \pi}{a}\right)^{2} \sin \left(\frac{m \pi x}{a}\right) \sin \left(\frac{n \pi y}{b}\right) \\
\left(\frac{\partial^{2} w(x, y)}{\partial y^{2}}\right) & =-\sum_{m=1}^{\infty} \sum_{n=1}^{\infty}\left(\frac{n \pi}{b}\right)^{2} \sin \left(\frac{m \pi x}{a}\right) \sin \left(\frac{n \pi y}{b}\right)
\end{aligned}
$$

$$
\left(\frac{\partial^{2} w(x, y)}{\partial x \partial y}\right)=\sum_{m=1}^{\infty} \sum_{n=1}^{\infty}\left(\frac{m \pi}{a}\right)\left(\frac{n \pi}{b}\right) \cos \left(\frac{m \pi x}{a}\right) \cos \left(\frac{n \pi y}{b}\right)
$$

Combining equation (38) through equation (40), finally, the general equation for SS plates without any edge condition can be written as

$$
\nabla^{4} F=-\left(\frac{E h^{2} \pi^{4} m^{2} n^{2}}{2 a^{2} b^{2}}\right)\left\{\sum_{m=1}^{\infty} \cos \left(\frac{2 m \pi x}{a}\right)+\sum_{n=1}^{\infty} \cos \left(\frac{2 n \pi y}{b}\right)\right\} .
$$

Equation (41) is a general solution which is the sum of complimentary function (CF) and particular integral (PI). Now, the PI in equation (41) is expressed as

$$
F_{1}=E h^{2}\left\{\eta_{1} \sum_{m=1}^{\infty} \cos \left(\frac{2 m \pi x}{a}\right)+\eta_{2} \sum_{n=1}^{\infty} \cos \left(\frac{2 n \pi y}{b}\right)\right\},
$$

wherein $\eta_{1}$ and $\eta_{2}$ are unknown constants and are determined by comparing equations (41) and (42), thus leading to

$$
\begin{aligned}
& \eta_{1}=-\left(\frac{a^{2}}{32 b^{2}}\right), \\
& \eta_{2}=-\left(\frac{b^{2}}{32 a^{2}}\right) .
\end{aligned}
$$

The PI of equation (41) is therefore further expressed as

$$
F_{1}=-\left(\frac{E h^{2}}{32}\right)\left(\left(\frac{a^{2}}{b^{2}}\right) \sum_{m=1}^{\infty} \cos \left(\frac{2 m \pi x}{a}\right)+\left(\frac{b^{2}}{a^{2}}\right) \sum_{n=1}^{\infty} \cos \left(\frac{2 n \pi y}{b}\right)\right) .
$$

Moreover, the CF in equation (41) is given by 


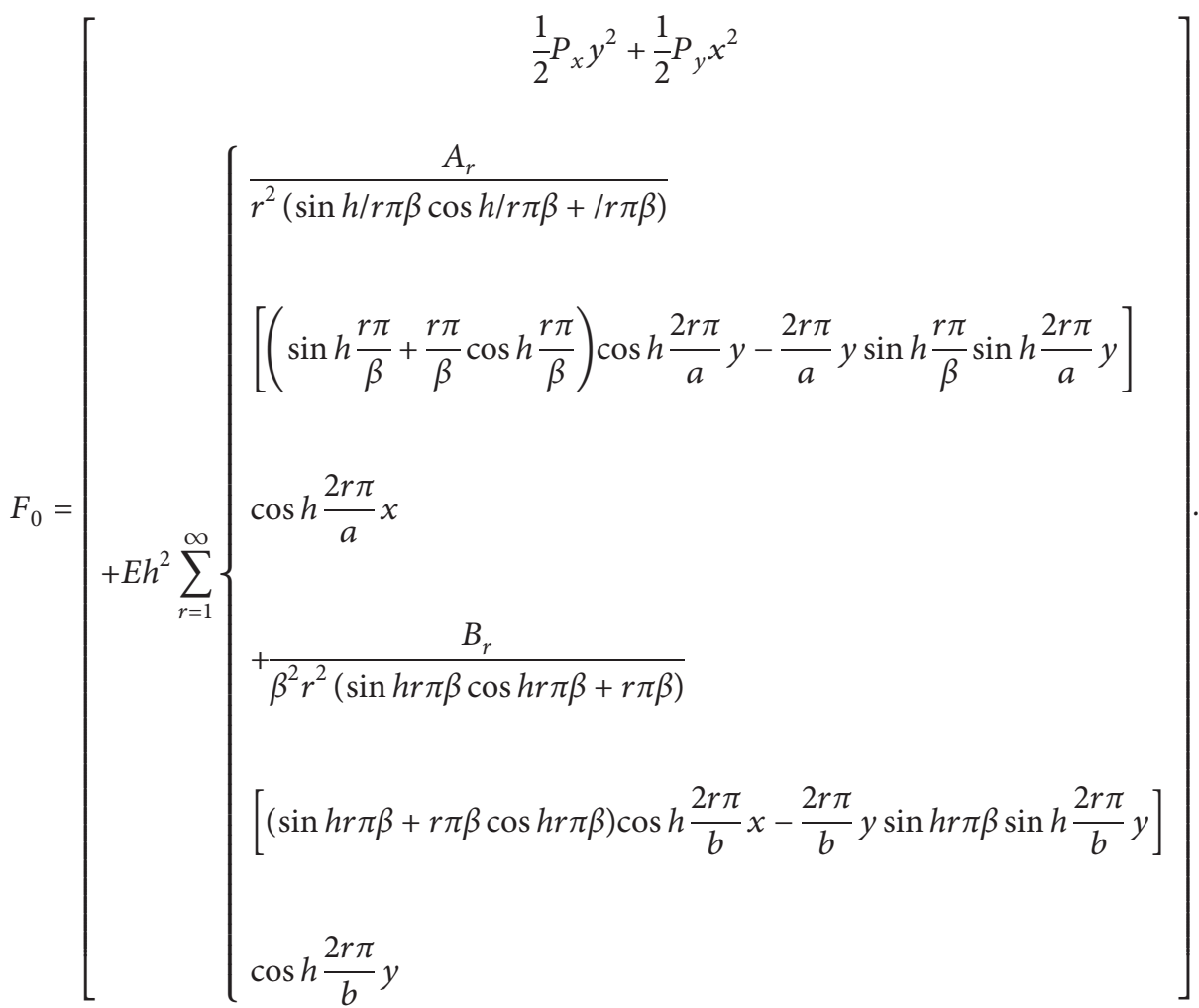

Therefore, in conclusion, the solution to equation (41) is expressed as

$$
\mathbf{F}=\mathbf{F}_{0}+\mathbf{F}_{1}
$$

It is important to notice that $P_{x}$ and $P_{y}$ denote tensile loads on the sides of a given thin rectangular plate of general size, varying from 0 to $a$ (in the $x$ direction) and from 0 to $b$ (in the $y$ direction), respectively. To derive the final expression for $P_{x}$ and $P_{y}$, it is assumed that all the edges of the plate remain straight after deformation.

This condition implies that the elongation of the plate along the $x$ direction is independent of $y$, and hence

$$
\begin{aligned}
\boldsymbol{\delta} \mathbf{x} & =\int_{0}^{\mathbf{a}}\left(\frac{\partial \mathbf{u}}{\partial \mathbf{x}}\right) \mathbf{d} \mathbf{x} \\
& =\int_{0}^{\mathbf{a}} \frac{1}{\mathbf{E}}\left(\left(\frac{\partial^{2} \mathbf{F}}{\partial \mathbf{y}^{2}}\right)-\boldsymbol{v}\left(\frac{\partial^{2} \mathbf{F}}{\partial \mathbf{x}^{2}}\right)\right)-\left(\frac{1}{2}\right)\left(\frac{\partial \mathbf{w}}{\partial \mathbf{x}}\right)^{2} \mathbf{d x} \\
\boldsymbol{\delta} \mathbf{x} & =\int_{0}^{\mathbf{a}}\left(\frac{1}{\mathbf{E}}\right)\left(\frac{\partial^{2} \mathbf{F}}{\partial \mathbf{y}^{2}}\right)-\boldsymbol{v} \int_{\mathbf{a}}^{\mathbf{a}}\left(\frac{1}{\mathbf{E}}\right)\left(\frac{\partial^{2} \mathbf{F}}{\partial \mathbf{x}^{2}}\right)-\int_{0}^{\mathbf{a}}\left(\frac{1}{2}\right)\left(\frac{\partial \mathbf{w}}{\partial \mathbf{x}}\right)^{2}
\end{aligned}
$$

Herein,

$$
\begin{aligned}
& \int_{0}^{a} \frac{1}{E}\left(\frac{\partial^{2} F}{\partial y^{2}}\right)=\left(\frac{1}{E h^{2}}\right) \int_{0}^{a}\left(P_{x}-\left(\left(\frac{n \pi}{b}\right)^{2} \sum_{m=1}^{\infty} \sin \left(\frac{m \pi x}{a}\right) \sum_{n=1}^{\infty} \sin \left(\frac{n \pi y}{b}\right)\right)\right) \mathrm{d} x, \\
& \int_{0}^{a} \frac{1}{E}\left(\frac{\partial^{2} F}{\partial y^{2}}\right)=\frac{1}{E}\left(P_{x} x-\left(\left(\frac{n \pi}{b}\right)^{2}\left(\frac{a}{m \pi}\right) \sum_{n=1}^{\infty} \cos \left(\frac{m \pi x}{a}\right) \sum_{n=1}^{\infty} \sin \left(\frac{n \pi y}{b}\right)\right)\right)_{0}^{a} .
\end{aligned}
$$

After applying the above limits and solving equation (48), it is found that

$$
\boldsymbol{\delta} \mathbf{x}=\frac{\mathbf{P}_{\mathbf{x}} \mathbf{a}}{\mathbf{E h}^{2}}-\boldsymbol{v}\left(\frac{\mathbf{P}_{y} \mathbf{a}}{\mathbf{E h}^{2}}\right)-\sum_{\mathbf{m}=1}^{\infty} \frac{\mathbf{m}^{2} \pi^{2}}{8 \mathbf{a}}
$$

Similarly, it is found that

$$
\boldsymbol{\delta} \mathbf{y}=\frac{\mathbf{P}_{y} \mathbf{b}}{\mathbf{E h}^{2}}-\mathbf{v}\left(\frac{\mathbf{P}_{\mathbf{x}} \mathbf{b}}{\mathbf{E h}^{2}}\right)-\sum_{\mathbf{n}=1}^{\infty} \frac{\mathbf{n}^{2} \pi^{2}}{8 \mathbf{b}} .
$$

Accordingly, to obtain the final solution, a final set of inplane force boundary conditions is used (i.e., all the edges are 
immovably constrained). Such an assumption implies that the elongation along $x$ and $y$ directions is zero, and thus

$$
\begin{aligned}
& P_{x}=\frac{\pi^{2} E h^{2}}{8 a^{2}}\left[\frac{\sum_{m=1}^{\infty} m^{2}+v^{2} \beta^{2} \sum_{n=1}^{\infty} n^{2}}{1-v^{2}}\right], \\
& P_{y}=\frac{\pi^{2} E h^{2}}{8 a^{2}}\left[\frac{\sum_{n=1}^{\infty} n^{2} \beta^{2}+v \sum_{m=1}^{\infty} m^{2}}{1-v^{2}}\right] .
\end{aligned}
$$

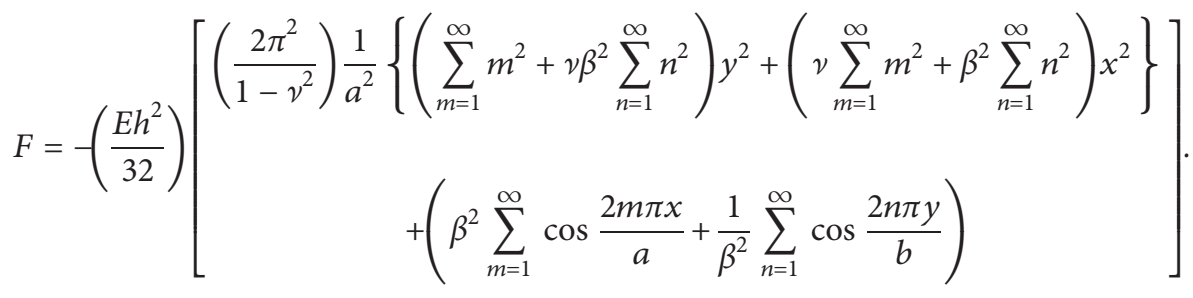

Now, assume that the function $F_{0}$ for stress-free edge condition is expressed as given in [21]:

$$
\begin{aligned}
& \bar{\varphi}_{p q}=\left(\begin{array}{c}
\frac{4 \beta}{\pi\left(p^{2}+\beta^{2} q^{2}\right)^{2}} \\
\times\left[\frac{p(-1)^{q} \varepsilon_{q} \sin h^{2}(p \pi / \beta)}{\sin h(p \pi / \beta) \cos h(p \pi / \beta)+(p \pi / \beta)} A_{p}+\frac{q(-1)^{p} \varepsilon_{p} \sin h^{2}(q \pi \beta)}{\sin h(q \pi \beta) \cos h(q \pi \beta)+(q \pi \beta)} B_{q}\right]
\end{array}\right), \\
& \varepsilon_{p}=\left\{\begin{array}{l}
0.5, p=0, \\
1.0, p>0,
\end{array}\right. \\
& \varepsilon_{q}=\left\{\begin{array}{l}
0.5, q=0 \\
1.0, q>0
\end{array}\right.
\end{aligned}
$$

The coefficients $A_{p}(p=0,1)$ and $B_{q}(q=0,1)$ are the solutions of the linear systems. 
It is to be noted that the function $F_{0}$ for SS plates and movable edges is equal to zero.
For easy understanding, equation (1) is now expressed as $\mathrm{I}(\mathrm{w}, \mathrm{F})$. The solutions for the membrane stiffness of the plate are detected as

$$
\begin{aligned}
& \int_{0}^{a} \int_{0}^{b} I(w, F) \sin \left(\frac{m \pi x}{a}\right) \sin \left(\frac{n \pi y}{b}\right) \mathrm{d} x \mathrm{~d} y \\
& =\int_{0}^{a} \int_{0}^{b}\left\{\left(\begin{array}{c}
\rho h\left(\frac{\partial^{2} w}{\partial t^{2}}\right)+\left(\frac{E h^{3}}{12\left(1-v^{2}\right)}\right) \nabla^{2} w \\
-h\left(\left(\frac{\partial^{2} F}{\partial y^{2}}\right)\left(\frac{\partial^{2} w}{\partial x^{2}}\right)+\left(\frac{\partial^{2} F}{\partial x^{2}}\right)\left(\frac{\partial^{2} w}{\partial y^{2}}\right)\right)- \\
2\left(\frac{\partial^{2} F}{\partial x \partial y}\right)\left(\frac{\partial^{2} w}{\partial x \partial y}\right)-q(x, y, t)
\end{array}\right) \sin \left(\frac{m \pi x}{a}\right) \sin \left(\frac{n \pi y}{b}\right)\right\} \mathrm{d} x \mathrm{~d} y=0 .
\end{aligned}
$$

Employing first the Ritz-Galerkin method for the solution of equation (58), in particular, the bending stiffness $K_{1}$ is determined as

$$
K_{1}=\left(\frac{\pi^{4} D a b}{4}\right) \sum_{m=1}^{\infty} \sum_{n=1}^{\infty}\left[\left(\frac{m}{a}\right)^{2}+\left(\frac{n}{b}\right)^{2}\right]^{2} .
$$

Moreover, the membrane stiffness $K_{3}$ (having a fundamental role for the nonlinear behavior of the examined plates) is defined as

$$
K_{3}=-h\left(\left(\frac{\partial^{2} F}{\partial y^{2}}\right)\left(\frac{\partial^{2} w}{\partial x^{2}}\right)+\left(\frac{\partial^{2} F}{\partial x^{2}}\right)\left(\frac{\partial^{2} w}{\partial y^{2}}\right)-2\left(\frac{\partial^{2} F}{\partial x \partial y}\right)\left(\frac{\partial^{2} w}{\partial x \partial y}\right)\right) \sin \left(\frac{m \pi x}{a}\right) \sin \left(\frac{n \pi y}{b}\right)
$$

Solving equation (57), $K_{3}$ can be therefore expressed for a SS plate with immovably constrained edge as

$$
\begin{aligned}
K_{3}= & \left(\frac{a b}{4}\right)\left(\frac{\pi^{4} E h^{3}}{8 a^{4}}\right) \\
& {\left[\begin{array}{c}
\sum_{n=1}^{\infty} n^{4} \beta^{4}+2 \nu \beta^{2} \sum_{m=1}^{\infty} \sum_{n=1}^{\infty} m^{2} n^{2}+\sum_{m=1}^{\infty} m^{4} \\
+\frac{1}{2}\left(1+\left(\frac{a^{4}}{b^{4}}\right)\right) \sum_{m=1}^{\infty} \sum_{n=1}^{\infty} m^{2} n^{2}
\end{array}\right] . }
\end{aligned}
$$

Similarly, for the membrane stress-free edge condition, $K_{3}$ is defined as

$$
\begin{aligned}
K_{3}= & \left(\frac{a b}{4}\right)\left(\frac{2 \pi^{4} E h^{3}}{a^{2} b^{2}}\right) \\
& {\left[\bar{\varphi}_{01}+\bar{\varphi}_{10}-\left(\frac{1}{32}\right)\left(\left(\frac{a^{2}}{b^{2}}\right)+\left(\frac{b^{2}}{a^{2}}\right)\right)\right] f^{3} . }
\end{aligned}
$$

For the movable edge condition, finally, the membrane stiffness $K_{3}$ is defined as

$$
K_{3}=\left(\frac{a b}{4}\right)\left(\frac{\pi^{4} E h^{3}}{16 a^{4}}\right)\left(1+\left(\frac{a^{4}}{b^{4}}\right)\right) \sum_{m=1}^{\infty} \sum_{n=1}^{\infty} m^{2} n^{2} .
$$

Now, for the force $P(t)$, it is assumed that

$$
P(t)=\left(\frac{4 a b}{(\pi)^{2}}\right) \sum_{m=1,3,5, \ldots} \sum_{n=1,3,5, \ldots}\left(\frac{1}{m n}\right) P_{1}(t) \mathrm{d} t .
$$

Further, the bending strains in the $x$ and $y$ directions are defined as

$$
\begin{aligned}
& \boldsymbol{\varepsilon}_{\mathrm{xx}}=\frac{1}{\mathbf{E}}\left[\frac{\partial^{2} \mathbf{F}}{\partial \mathbf{y}^{2}}-\boldsymbol{v} \frac{\partial^{2} \mathbf{F}}{\partial \mathbf{x}^{2}}\right], \\
& \boldsymbol{\varepsilon}_{\mathrm{yy}}=\frac{1}{\mathbf{E}}\left[\frac{\partial^{2} \mathbf{F}}{\partial \mathbf{x}^{2}}-\boldsymbol{v} \frac{\partial^{2} \mathbf{F}}{\partial \mathbf{y}^{2}}\right] .
\end{aligned}
$$

The shear strains in the $x-y$ direction are zero, due to assumed function, and such an assumption is in close 
correlation with various literature researchers (see, for example, $[21,26])$. This means that

$$
\gamma_{x y}=-\left(\frac{2(1+\nu)}{E}\right)\left(\frac{\partial^{2} F}{\partial x \partial y}\right)=0 .
$$

$$
\varepsilon_{x x}=\left(\frac{h^{2}}{32}\right)\left(\frac{4 \pi^{2}}{a^{2}}\right)\left[\left[\left(\sum_{m=1}^{\infty} m^{2}\right)+\sum_{n=1}^{\infty} n^{2} \cos \left(\frac{2 n \pi y}{b}\right)\right]-\left[\nu \beta^{2} \sum_{m=1}^{\infty} m^{2} \cos \left(\frac{2 m \pi x}{a}\right)\right]\right] .
$$

Assuming that $m=1$ and $n=1$, the latter results in

For a square plate $(\mathrm{a} / \mathrm{b}=1)$, it is therefore found that

$$
\varepsilon_{\mathrm{xx}}=\left(\frac{h^{2} \pi^{2}}{8 a^{2}}\right)\left[1+\cos \left(\frac{2 \pi y}{b}\right)-\nu \beta^{2} \cos \left(\frac{2 \pi x}{a}\right)\right] \text {. }
$$

Finally, the strains in $x$ direction are therefore defined as

$$
\varepsilon_{x x}=\left(\frac{h^{2} \pi^{2}}{8 a^{2}}\right)\left[1+\cos \left(\frac{2 \pi y}{a}\right)-v \cos \left(\frac{2 \pi x}{a}\right)\right] .
$$

It is noteworthy that the above equation as herein derived by using infinite trigonometric series equals the strain equation given by Bauer [25] for square plates.

Similarly, the strain along the $y$ direction is defined as

$$
\left.\varepsilon_{y y}=\left(\frac{1}{E}\right)\left(\frac{E h^{2}}{32}\right)\right]\left[\begin{array}{c}
{\left[\begin{array}{c}
\left(\frac{2 \pi^{2}}{1-v^{2}}\right) \frac{1}{a^{2}}\left(\nu \sum_{m=1}^{\infty} m^{2}+\beta^{2} \sum_{n=1}^{\infty} n^{2}\right) \times 2 \\
+\beta^{2}\left(\frac{2 m \pi}{a}\right)^{2} \sum_{m=1}^{\infty} \cos \frac{2 m \pi x}{a}
\end{array}\right]} \\
\left.-\nu\left[\begin{array}{c}
\left(\frac{2 \pi^{2}}{1-v^{2}}\right) \frac{1}{a^{2}}\left(\sum_{m=1}^{\infty} m^{2}+v \beta^{2} \sum_{n=1}^{\infty} n^{2}\right) \\
\times 2+\frac{1}{\beta^{2}}\left(\frac{2 n \pi}{b}\right)^{2} \sum_{n=1}^{\infty} \cos \left(\frac{2 n \pi y}{b}\right)
\end{array}\right]\right]
\end{array}\right] .
$$

Again, assuming $m=1$ and $n=1$, it is found that

$$
\varepsilon_{y y}=\left(\frac{h^{2} \pi^{2}}{8 b^{2}}\right)\left[1+\cos \left(\frac{2 \pi x}{a}\right)-\left(\frac{v}{\beta^{2}}\right) \cos \left(\frac{2 \pi y}{b}\right)\right] \text {. }
$$

Therefore, for a square plate,

$$
\varepsilon_{y y}=\left(\frac{h^{2} \pi^{2}}{8 a^{2}}\right)\left[1+\cos \left(\frac{2 \pi x}{a}\right)-\nu \cos \left(\frac{2 \pi y}{a}\right)\right],
$$

or

$$
\varepsilon_{y y}=\left(\frac{h^{2} \pi^{2}}{8 b^{2}}\right)\left[1+\cos \left(\frac{2 \pi x}{b}\right)-v \cos \left(\frac{2 \pi y}{b}\right)\right]
$$

and equation (70) coincides with the strain equation given by Bauer [25] for square plates.

Now, merging all the so-obtained expressions in equation (55), it is found that 


$$
\int_{0}^{a} \int_{0}^{b} I(w, F) \sin \left(\frac{m \pi x}{a}\right) \sin \left(\frac{n \pi y}{b}\right) \mathrm{d} x \mathrm{~d} y=\left\{\begin{array}{c}
\rho h^{2} \ddot{f}+\sum_{m=1}^{\infty} \sum_{n=1}^{\infty}\left\{\left(\frac{m \pi}{a}\right)^{4}+\left(\frac{n \pi}{b}\right)^{4}+2\left(\frac{m \pi}{a}\right)^{2}\left(\frac{n \pi}{b}\right)^{2}\right\} D h f- \\
\left(\frac{4}{\pi^{2}}\right) \sum_{m=1}^{\infty} \sum_{n=1}^{\infty} \frac{1-\cos (m \pi)-\cos (n \pi)+\cos (m \pi) \cos (n \pi)}{m n} \\
+\frac{\pi^{4} E h^{4}}{8 a^{4}}\left[\frac{\sum_{n=1}^{\infty} n^{4} \beta^{4}+2 \nu \beta^{2} \sum_{m=1}^{\infty} \sum_{n=1}^{\infty} m^{2} n^{2}+\sum_{m=1}^{\infty} m^{4}}{1-v^{2}}+\left(\frac{1}{2}\right)\left(1+\left(\frac{a^{4}}{b^{4}}\right)\right) \sum_{m=1}^{\infty} \sum_{n=1}^{\infty} m^{2} n^{2}\right] f^{3}
\end{array}\right\} .
$$

Substituting $m=1$ and $n=1$ in equation (71), the solution is therefore obtained for various boundary conditions.
The solution for a SS plate with immovably constrained edges, in particular, is given by

$$
\left\{\begin{array}{c}
\rho h^{2} \ddot{f}+D h\left\{\left(\frac{\pi}{a}\right)^{4}+\left(\frac{\pi}{b}\right)^{4}+2\left(\frac{\pi}{a}\right)^{2}\left(\frac{\pi}{b}\right)^{2}\right\} f+ \\
\frac{\pi^{4} E h^{4}}{8 a^{4}}\left[\frac{1+2 \nu\left(a^{2} / b^{2}\right)+\left(a^{4} / b^{4}\right)}{1-v^{2}}+\frac{1}{2}\left(1+\left(\frac{a^{4}}{b^{4}}\right)\right)\right] f^{3}
\end{array}\right\}=\frac{16}{\pi^{2}}
$$

For a SS plate with movable edges, it is

$$
\left\{\begin{array}{c}
\rho h^{2} \ddot{f}+\sum_{m=1}^{\infty} \sum_{n=1}^{\infty}\left\{\left(\frac{m \pi}{a}\right)^{4}+\left(\frac{n \pi}{b}\right)^{4}+2\left(\frac{m \pi}{a}\right)^{2}\left(\frac{n \pi}{b}\right)^{2}\right\} D h f- \\
\frac{4}{\pi^{2}} \sum_{m=1}^{\infty} \sum_{n=1}^{\infty} \frac{1-\cos (m \pi)-\cos (n \pi)+\cos (m \pi) \cos (n \pi)}{m n} \\
+\frac{\pi^{4} E h^{4}}{8 a^{4}}\left[\frac{1}{2}\left(1+\left(\frac{a^{4}}{b^{4}}\right)\right) \sum_{m=1}^{\infty} \sum_{n=1}^{\infty} m^{2} n^{2}\right] f^{3}
\end{array}\right\}=0
$$

The solution for a SS plate with movable constrained edges is

$$
\left\{\begin{array}{c}
\rho h^{2} \ddot{f}+\operatorname{Dh}\left\{\left(\frac{\pi}{a}\right)^{4}+\left(\frac{\pi}{b}\right)^{4}+2\left(\frac{\pi}{a}\right)^{2}\left(\frac{\pi}{b}\right)^{2}\right\} f \\
-\frac{\pi^{4} E h^{4}}{8 a^{4}}\left[\frac{1}{2}\left(1+\left(\frac{a^{4}}{b^{4}}\right)\right)\right] f^{3}
\end{array}\right\}=\frac{16}{\pi^{2}}
$$

Finally, the solution for the SS plate with stress-free edges is given by

$$
\left\{\begin{array}{c}
\rho h^{2} \ddot{f}+D h\left\{\left(\frac{\pi}{a}\right)^{4}+\left(\frac{\pi}{b}\right)^{4}+2\left(\frac{\pi}{a}\right)^{2}\left(\frac{\pi}{b}\right)^{2}\right\} f-\left(\frac{2 \pi^{4} E h^{4}}{a^{2} b^{2}}\right) \\
{\left[\varphi_{01}^{\prime}+\varphi_{10}^{\prime}-\frac{1}{32}\left(\frac{a^{2}}{b^{2}}+\frac{b^{2}}{a^{2}}\right)\right] f^{3}}
\end{array}\right\}=\frac{16}{\pi^{2}} .
$$


4.2. Clamped (CC) Plate. Assuming the deflection shape function for a thin elastic plate under blast loading as per equation (24), the reference function satisfies all the boundary conditions. Here, the mass $m$ is defined by equation (27) and can be further expressed as

$$
m=\frac{\rho a b h}{16 a b} \int_{0}^{a} \int_{0}^{b}\left(1-2 \cos \left(\frac{2 m \pi x}{a}\right)+\frac{1}{2}\left(1+\cos \left(\frac{4 m \pi x}{a}\right)\right) \times\left(1-2 \cos \frac{2 n \pi y}{b}+\frac{1}{2}\left(1+\cos \frac{4 n \pi y}{b}\right)\right) d x d y .\right.
$$

Integrating the above equation, it reduces to

$$
m=\frac{\rho a b h}{16 a b}\left\{\left[\begin{array}{c}
\left.(x-2) \frac{\sin (2 m \pi x / a)}{(2 m \pi / a)}+\frac{1}{2}\left(x+\frac{\sin (4 m \pi x / a)}{(4 m \pi / a)}\right)\right) \\
\left(y-2 \frac{\sin (2 n \pi y / b)}{(2 n \pi / b)}+\frac{1}{2}\left(y+\frac{\sin (4 n \pi y / b)}{(4 n \pi / b)}\right)\right)
\end{array}\right]_{0}^{a}\right\}_{0}^{b}
$$

Applying the limits, it is found that

$$
m=\left(\frac{9 \rho a b h}{64}\right)
$$

where the latter is suitable for a rectangular, elastic isotropic plate with dimensions $a \times b$, thickness $h$, and density $\rho$, under a CC condition. Further, the external normal force per unit area of the plate, $P$, is expressed by equation (30). As mentioned earlier for the SS plate, in the case of uniform lateral pressure, it is $Q(x, y)=1$; therefore,

$$
P(t)=\int_{0}^{a} \int_{0}^{b} \sum_{m=1}^{\infty} \sum_{n=1}^{\infty} \sin ^{2}\left(\frac{m \pi x}{a}\right) \sin ^{2}\left(\frac{n \pi y}{b}\right) \mathrm{d} x \mathrm{~d} y P_{1}(t) \mathrm{d} t
$$

or

$$
P(t)=\frac{1}{4} \int_{0}^{a} \int_{0}^{b} \sum_{m=1}^{\infty} \sum_{n=1}^{\infty}\left(1-\cos \frac{2 m \pi x}{a}\right)\left(1-\cos \frac{2 n \pi y}{b}\right) \mathrm{d} x \mathrm{~d} y P_{1}(t) \mathrm{d} t .
$$

Integrating the above equation, it reduces to

$$
P(t)=\left(\frac{1}{4}\right)\left[\sum_{m=1}^{\infty} \sum_{n=1}^{\infty}\left(x-\frac{\sin (2 m \pi x / a)}{(2 m \pi / a)}\right)\left(y-\frac{\sin (2 n \pi y / b)}{(2 n \pi / b)}\right)_{0}^{a}\right]_{0}^{b} P_{1}(t) \mathrm{d} t
$$

Applying the limits, we get

$$
P(t)=\left(\frac{a b}{4}\right) P_{1}(t)
$$

where the latter represents the external normal force per unit area of the plate, $P$, in trigonometric series (and is substantially different from the proposal in [33]). 
Now, the bending stiffness $K_{1}$ of the plate is expressed as

$$
K_{1}=D \int_{0}^{a} \int_{0}^{b}\left\{\begin{array}{c}
{\left[\frac{\partial^{2} \varphi(x, y)}{\partial x^{2}}+\frac{\partial^{2} \varphi(x, y)}{\partial y^{2}}\right]^{2}} \\
-2(1-v)\left[\frac{\partial^{2} \varphi(x, y)}{\partial x^{2}} \frac{\partial^{2} \varphi(x, y)}{\partial y^{2}}-\left[\frac{\partial^{2} \varphi(x, y)}{\partial x \partial y}\right]^{2}\right]
\end{array}\right\} \mathrm{d} x \mathrm{~d} y
$$

Applying the assumed deflection function and solving equation (87), $K_{1}$ for a CC plate is therefore found in

$$
K_{1}=\left(\frac{D \pi^{4} a b}{4}\right) \sum_{m=1}^{\infty} \sum_{n=1}^{\infty}\left[\left(\frac{3 m^{4}}{a^{4}}\right)+\left(\frac{2 m^{2} n^{2}}{a^{2} b^{2}}\right)+\left(\frac{3 n^{4}}{b^{4}}\right)\right] \text {. }
$$

In order to obtain the membrane stiffness function for large deflections of rectangular CC plates, the substitution of deflection (i.e., equations (23) and (25) for the CC case) into equation (2) is first taken into account, so as to achieve a suitable stress function.

This yields to

$$
\nabla^{4} F_{1}=\left(\frac{\pi^{4} m^{2} n^{2}}{2 a^{2} b^{2}}\right)\left[\begin{array}{c}
\cos \left(\frac{2 m \pi x}{a}\right)+\cos \left(\frac{2 n \pi y}{b}\right)+ \\
\cos \left(\frac{2 n \pi y}{b}\right) \cos \left(\frac{4 m \pi x}{a}\right)-\cos \left(\frac{4 m \pi x}{a}\right) \\
+\cos \left(\frac{4 n \pi y}{b}\right) \cos \left(\frac{2 m \pi x}{a}\right)-\cos \left(\frac{4 m \pi x}{a}\right) \\
-2 \cos \left(\frac{2 n \pi y}{b}\right) \cos \left(\frac{2 m \pi x}{a}\right)
\end{array}\right] .
$$

The solution of equation (85) takes the form of CF and PI parts, wherein PI is given by

$$
F_{1}=\frac{1}{32}\left[\begin{array}{c}
\frac{n^{2} \beta^{2}}{m^{2}} \cos \left(\frac{2 m \pi x}{a}\right)+\frac{m^{2}}{n^{2} \beta^{2}} \cos \left(\frac{2 n \pi y}{b}\right)+ \\
\frac{m^{2} n^{2} \beta^{2}}{\left(4 m^{2}+n^{2} \beta^{2}\right)^{2}} \cos \left(\frac{2 n \pi y}{b}\right) \cos \left(\frac{4 m \pi x}{a}\right)+ \\
\frac{m^{2} n^{2} \beta^{2}}{\left(m^{2}+4 n^{2} \beta^{2}\right)^{2}} \cos \left(\frac{4 n \pi y}{b}\right) \cos \left(\frac{2 m \pi x}{a}\right)-\frac{n^{2} \beta^{2}}{16 m^{2}} \cos \left(\frac{4 m \pi x}{a}\right) \\
-\frac{m^{2}}{16 n^{2} \beta^{2}} \cos \left(\frac{4 m \pi x}{a}\right)-\frac{m^{2} n^{2} \beta^{2}}{\left(m^{2}+n^{2} \beta^{2}\right)^{2}} \cos \left(\frac{2 n \pi y}{b}\right) \cos \left(\frac{2 m \pi x}{a}\right)
\end{array}\right] .
$$

Therefore, the general solution is $F=F_{0}+F_{1}$, where

$$
F_{0}=\left(\frac{P_{x} y^{2}}{2}\right)+\left(\frac{P_{y} x^{2}}{2}\right)
$$


and $F_{0}$ for the stress-free edge condition is expressed as [21]

$$
\varphi_{p q}=\frac{4 \beta}{\pi\left(p^{2}+\beta^{2} q^{2}\right)^{2}} \times\left[\frac{p(-1)^{q} \varepsilon_{q} \sin h^{2}(p \pi / \beta)}{\sin h(p \pi / \beta) \cos h(p \pi / \beta)+(p \pi / \beta)} A_{p}+\frac{q(-1)^{p} \varepsilon_{p} \sin h^{2}(q \pi \beta)}{\sin h(q \pi \beta) \cos h(q \pi \beta)+(q \pi \beta)} B_{q}\right] .
$$

Moreover:

$$
\begin{aligned}
& \varepsilon_{p}=\left\{\begin{array}{l}
0.5, p=0, \\
1.0, p>0,
\end{array}\right. \\
& \varepsilon_{q}=\left\{\begin{array}{l}
0.5, q=0, \\
1.0, q>0,
\end{array}\right.
\end{aligned}
$$

while the coefficients $A_{p}$ and $B_{q}$ in equation (88) are the solutions of the linear systems.

To derive the expression for $P_{x}$ and $P_{y}$, all the edges of the plate are assumed to remain straight after the deformation. Following the same procedure earlier adopted for the SS plate, but with the reference CC function and boundary condition, it is found that

$$
\begin{gathered}
\boldsymbol{\delta} \mathbf{x}=\frac{\mathbf{P}_{\mathbf{x}} \mathbf{a}}{\mathbf{E h}^{2}}-\boldsymbol{v} \frac{\mathbf{P}_{\mathbf{y}} \mathbf{a}}{\mathbf{E h}^{2}}-\frac{3 E \mathbf{h}^{2}}{32} \sum_{m=1}^{\infty} \frac{\mathbf{m}^{2} \pi^{2}}{\mathbf{a}^{2}}=0, \\
\boldsymbol{\delta} \mathbf{y}=\frac{\mathbf{P}_{\mathbf{y}} \mathbf{b}}{\mathbf{E h}^{2}}-\boldsymbol{v} \frac{\mathbf{P}_{\mathbf{x}} \mathbf{b}}{\mathbf{E h}^{2}}-\frac{3 E \mathbf{h}^{2}}{32} \sum_{n=1}^{\infty} \frac{\mathbf{n}^{2} \pi^{2}}{\mathbf{b}^{2}}=0 .
\end{gathered}
$$

The final solution is found for the immovably constrained edges, given that the elongation along the $x$ and $y$ directions is zero:

$$
\begin{aligned}
& P_{x}=\frac{3 \pi^{2} E h^{2}}{32 a^{2}}\left[\frac{\sum_{n=1}^{\infty} m^{2}+v \sum_{m=1}^{\infty} n^{2} \beta^{2}}{1-v^{2}}\right], \\
& P_{y}=\frac{3 \pi^{2} E h^{2}}{32 a^{2}}\left[\frac{\sum_{n=1}^{\infty} n^{2} \beta^{2}+v \sum_{m=1}^{\infty} m^{2}}{1-v^{2}}\right] .
\end{aligned}
$$

Finally, the stress function for a CC plate with immovable edge conditions is defined as

$$
F=\left[\begin{array}{c}
\frac{3 \pi^{2} E h^{2}}{32 a^{2}}\left[\frac{\sum_{n=1}^{\infty} m^{2}+v \sum_{m=1}^{\infty} n^{2} \beta^{2}}{1-v^{2}}\right] \frac{y^{2}}{2}+\left(\frac{3 \pi^{2} E h^{2}}{32 a^{2}}\right)\left[\frac{\sum_{n=1}^{\infty} n^{2} \beta^{2}+v \sum_{m=1}^{\infty} m^{2}}{1-v^{2}}\right] \frac{x^{2}}{2} \\
+\frac{1}{32}\left[\begin{array}{c}
\left.\frac{n^{2} \beta^{2}}{m^{2}}\right) \cos \left(\frac{2 m \pi x}{a}\right)+\frac{m^{2}}{n^{2} \beta^{2}} \cos \left(\frac{2 n \pi y}{b}\right)+ \\
\frac{m^{2} n^{2} \beta^{2}}{\left(4 m^{2}+n^{2} \beta^{2}\right)^{2}} \cos \left(\frac{2 n \pi y}{b}\right) \cos \left(\frac{4 m \pi x}{a}\right)+ \\
\frac{m^{2} n^{2} \beta^{2}}{\left(m^{2}+4 n^{2} \beta^{2}\right)^{2}} \cos \left(\frac{4 n \pi y}{b}\right) \cos \left(\frac{2 m \pi x}{a}\right)-\frac{n^{2} \beta^{2}}{16 m^{2}} \cos \left(\frac{4 m \pi x}{a}\right) \\
-\frac{m^{2}}{16 n^{2} \beta^{2}} \cos \left(\frac{4 m \pi x}{a}\right)-\frac{m^{2} n^{2} \beta^{2}}{\left(m^{2}+n^{2} \beta^{2}\right)^{2}} \cos \left(\frac{2 n \pi y}{b}\right) \cos \left(\frac{2 m \pi x}{a}\right)
\end{array}\right]
\end{array}\right] .
$$

For easy understanding, as in the case of the SS plate, equation ( 1 ) is expressed as $\mathrm{I}(\mathrm{w}, \mathrm{F})$. The solution for the membrane stiffness of the plate is therefore derived as 


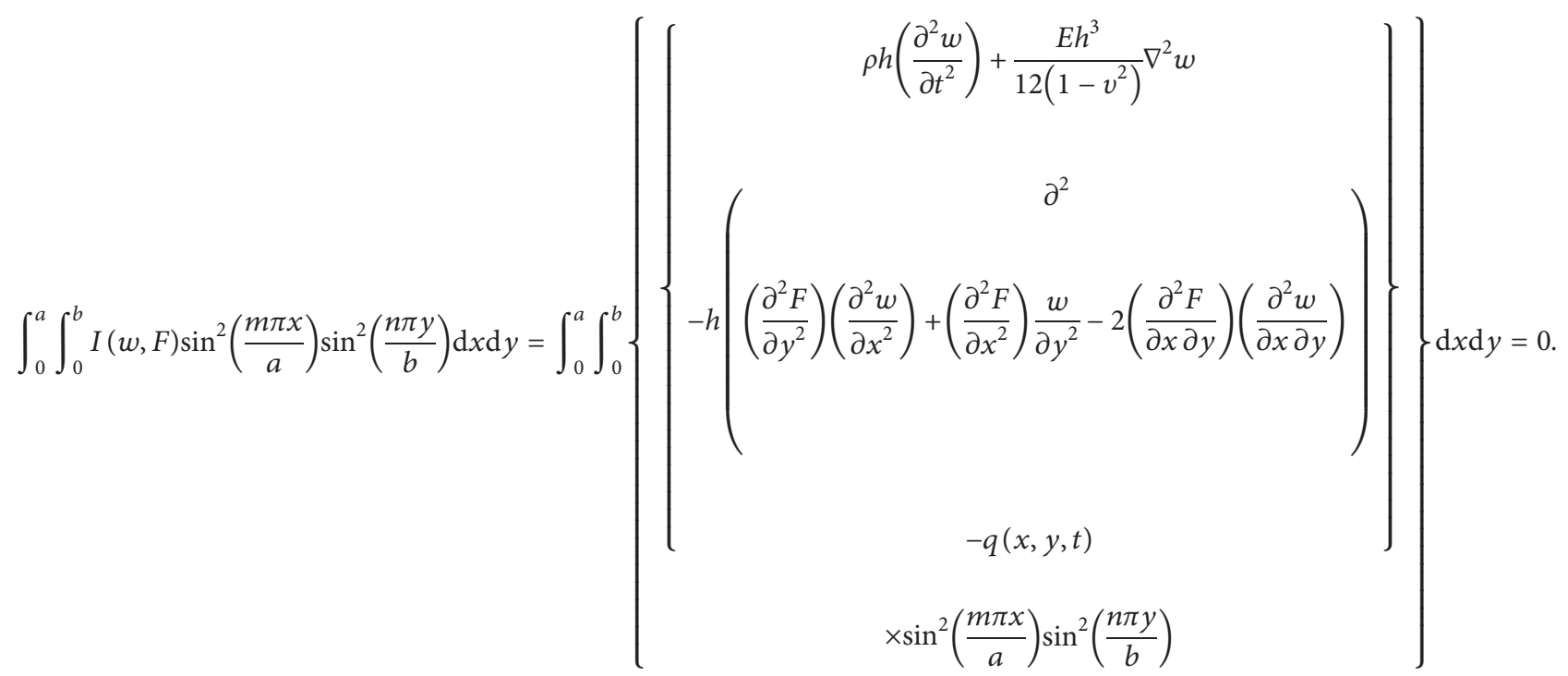

By employing the Ritz-Galerkin method to solve equation (53), the bending stiffness $K_{1}$ is first calculated as

$$
K_{1}=\frac{\pi^{4} D a b}{4} \sum_{m=1}^{\infty} \sum_{n=1}^{\infty}\left[\left(\frac{3 m^{4}}{a^{4}}\right)+\left(\frac{2 m^{2} n^{2}}{a^{2} b^{2}}\right)+\left(\frac{3 n^{4}}{b^{4}}\right)\right],
$$

where the mass is

$$
m=\left(\frac{9 \rho a b h}{64}\right) \text {. }
$$

The membrane stiffness $K_{3}$ for a CC plate is therefore obtained by applying the same methodology used for the SS plate.

For the immovably constrained edge condition, it is found that

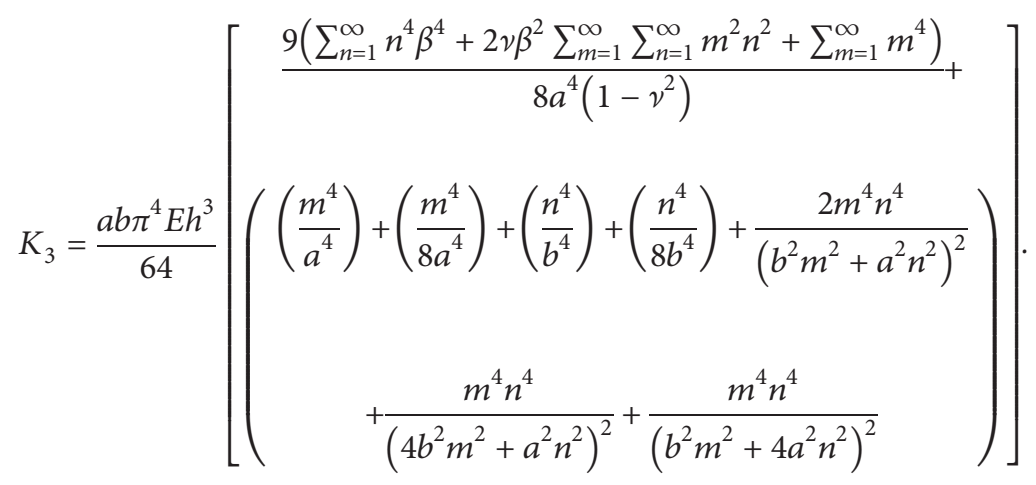

For the stress-free edge condition:

$$
K_{3}=\frac{a b \pi^{4} E h^{3}}{2 a^{2} b^{2}}\left(\begin{array}{c}
\bar{\varphi}_{01}+\bar{\varphi}_{10}+\bar{\varphi}_{11}+\frac{1}{2} \bar{\varphi}_{12}+\frac{1}{2} \bar{\varphi}_{21}+\bar{\varphi}_{02}+\bar{\varphi}_{20} \\
-\left(\frac{1}{32}\right)\left(\left(\frac{a^{4}}{b^{4}}\right)+\left(\frac{9}{8}\right)+\frac{2(a / b)^{4}}{\left(1+\left(a^{2} / b^{2}\right)\right)^{2}}+\left(\frac{a^{4}}{8 b^{4}}\right)+\frac{(a / b)^{4}}{\left(1+4\left(a^{2} / b^{2}\right)\right)^{2}}+\frac{(a / b)^{4}}{\left(4+\left(a^{2} / b^{2}\right)\right)^{2}}\right)
\end{array}\right) .
$$


For the movable edge condition, finally:

$$
K_{3}=\frac{a b \pi^{4} E h^{3}}{64 a^{2} b^{2}}\left(\left(\frac{a^{4}}{b^{4}}\right)+\left(\frac{9}{8}\right)+\left(\frac{2(a / b)^{4}}{\left(1+\left(a^{2} / b^{2}\right)\right)^{2}}\right)+\left(\frac{a^{4}}{8 b^{4}}\right)+\left(\frac{(a / b)^{4}}{\left(1+4\left(a^{2} / b^{2}\right)\right)^{2}}\right)+\left(\frac{(a / b)^{4}}{\left(4+\left(a^{2} / b^{2}\right)\right)^{2}}\right)\right)
$$

where the force $P(t)$ is given by

Therefore,

$$
P(t)=\left(\frac{a b}{4}\right) P_{1}(t)
$$

$$
\int_{0}^{a} \int_{0}^{b}\left(\frac{\partial^{2} F}{\partial x^{2}}\right)\left(\frac{\partial^{2} w}{\partial y^{2}}\right)=-\sum_{m=1}^{\infty} \sum_{n=1}^{\infty}\left[\begin{array}{l}
\left(\frac{3 a b \pi^{2} m^{2}}{16 a^{2}}\right)+\left(\frac{m^{2}}{n^{2} \beta^{2}} \times\left(\frac{2 n \pi}{b}\right)^{2}\left(\frac{m \pi}{a}\right)^{2}\left(\frac{a b}{8}\right)\right) \\
+\frac{1}{32}\left(\frac{n^{2} m^{2} \beta^{2}}{\left(4 m^{2}+n^{2} \beta^{2}\right)^{2}} \times\left(\frac{2 n \pi}{b}\right)^{2}\left(\frac{m \pi}{a}\right)^{2}\left(\frac{a b}{16}\right)\right)+ \\
\left.\left(m^{2}+4 n^{2} \beta^{2}\right)^{2} \times\left(\frac{4 n \pi}{b}\right)^{2}\left(\frac{m \pi}{a}\right)^{2}\left(\frac{a b}{32}\right)\right) \\
+\left(\frac{m^{2}}{16 n^{2} \beta^{2}} \times\left(\frac{4 n \pi}{b}\right)^{2}\left(\frac{m \pi}{a}\right)^{2}\left(\frac{a b}{32}\right)\right) \\
\left.+\left(m^{2}+n^{2} \beta^{2}\right)^{2} \times\left(\frac{2 n \pi}{b}\right)^{2}\left(\frac{m \pi}{a}\right)^{2}\left(\frac{a b}{8}\right)\right)
\end{array}\right],
$$

or 


$$
\int_{0}^{a} \int_{0}^{b}\left(\frac{\partial^{2} F}{\partial y^{2}}\right)\left(\frac{\partial^{2} w}{\partial x^{2}}\right)=-\frac{1}{32} \sum_{m=1}^{\infty} \sum_{n=1}^{\infty}\left[\begin{array}{l}
\left(\frac{6 a b \pi^{2} n^{2}}{b^{2}}\right)+\left(\frac{n^{2} \beta^{2}}{m^{2}} \times\left(\frac{n \pi}{b}\right)^{2}\left(\frac{2 m \pi}{a}\right)^{2}\left(\frac{a b}{8}\right)\right)+ \\
\left(\frac{n^{2} m^{2} \beta^{2}}{\left(4 m^{2}+n^{2} \beta^{2}\right)^{2}} \times\left(\frac{n \pi}{b}\right)^{2}\left(\frac{4 m \pi}{a}\right)^{2}\left(\frac{a b}{32}\right)\right)+ \\
\left(\frac{n^{2} m^{2} \beta^{2}}{\left(m^{2}+4 n^{2} \beta^{2}\right)^{2}} \times\left(\frac{n \pi}{b}\right)^{2}\left(\frac{2 m \pi}{a}\right)^{2}\left(\frac{a b}{16}\right)\right) \\
\left(\frac{n^{2} \beta^{2}}{16 m^{2}} \times\left(\frac{n \pi}{b}\right)^{2}\left(\frac{4 m \pi}{a}\right)^{2}\left(\frac{a b}{32}\right)\right)+ \\
\left(\frac{n^{2} m^{2} \beta^{2}}{\left(m^{2}+n^{2} \beta^{2}\right)^{2}} \times\left(\frac{n \pi}{b}\right)^{2}\left(\frac{2 m \pi}{a}\right)^{2}\left(\frac{a b}{8}\right)\right)
\end{array}\right] .
$$

Here, $\int_{0}^{b} \int_{0}^{a} 2\left(\partial^{2} F / \partial x \partial y\right)\left(\partial^{2} w / \partial x \partial y\right)=0$.

Finally, summing up the above expressions, it is found that

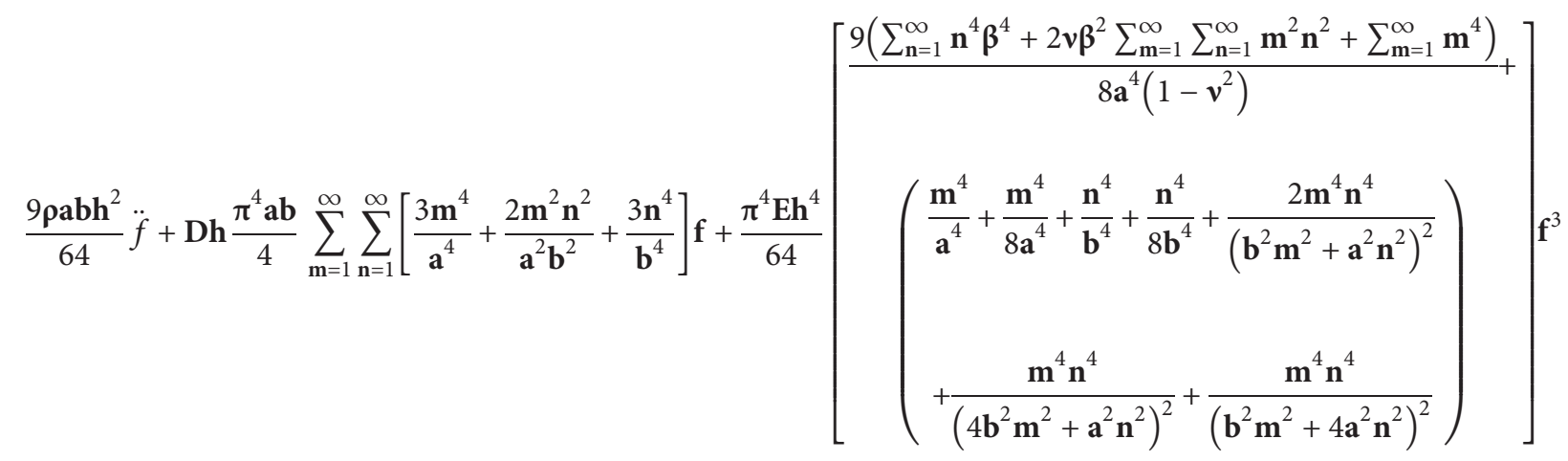$$
=\frac{\mathbf{a b}}{4} \mathbf{p}(\mathbf{t}) \text {. }
$$

By substitution of $m=1$ and $n=1$, the expression for different CC edge conditions can be therefore finally obtained.

The solution for a CC plate with immovable edges is given by 


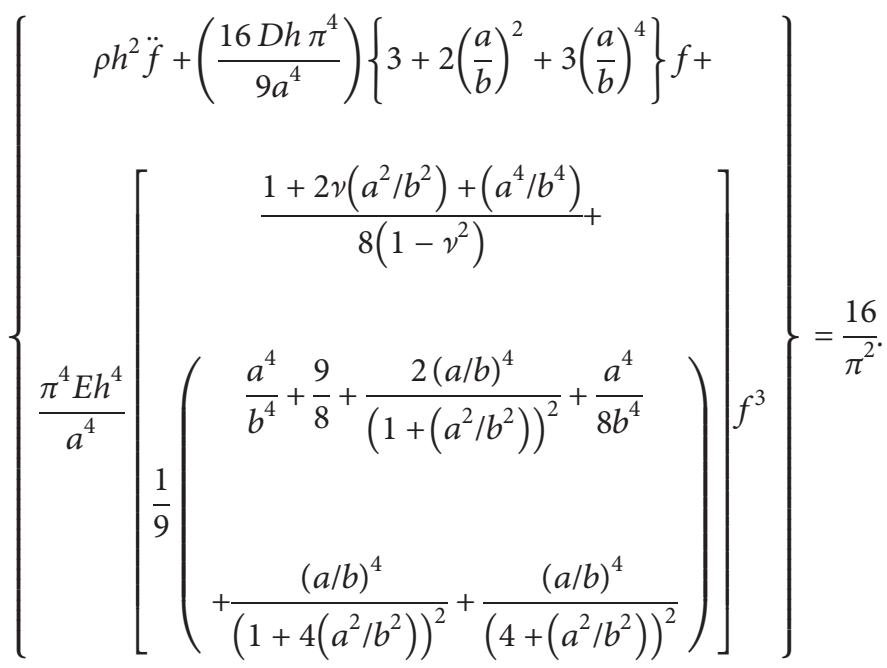

The solution for the CC plate with movable edges is

$$
\left\{\begin{array}{c}
\rho h^{2} \ddot{f}+\frac{D h \pi^{4}}{9 a^{4}}\left\{3+2\left(\frac{a}{b}\right)^{2}+3\left(\frac{a}{b}\right)^{4}\right\} f+ \\
\frac{\pi^{4} E h^{4}}{9 a^{2} b^{2}}\left(\begin{array}{c}
\frac{a^{4}}{b^{4}}+\frac{9}{8}+\frac{2(a / b)^{4}}{\left(1+\left(a^{2} / b^{2}\right)\right)^{2}}+\frac{a^{4}}{8 b^{4}} \\
+\frac{(a / b)^{4}}{\left(1+4\left(a^{2} / b^{2}\right)\right)^{2}}+\frac{(a / b)^{4}}{\left(4+\left(a^{2} / b^{2}\right)\right)^{2}}
\end{array}\right) f^{3}
\end{array}\right\}=\frac{16}{\pi^{2}}
$$

while the solution for the CC plate with stress-free edges is

$$
\left\{\begin{array}{c}
\rho h^{2} \ddot{f}+\frac{D h \pi^{4}}{9 a^{4}}\left\{3+2\left(\frac{a}{b}\right)^{2}+3\left(\frac{a}{b}\right)^{4}\right\} f+ \\
\frac{32 \pi^{4} E h^{4}}{9 a^{2} b^{2}}\left(\begin{array}{c}
\varphi_{01}^{\prime}+\varphi_{10}^{\prime}+\varphi_{11}^{\prime}+\frac{1}{2} \varphi_{12}^{\prime}+\frac{1}{2} \varphi_{21}^{\prime}+\varphi_{02}^{\prime}+\varphi_{20}^{\prime} \\
-\frac{1}{32}\left(\begin{array}{c}
\frac{a^{4}}{b^{4}}+\frac{9}{8}+\frac{2(a / b)^{4}}{\left(1+\left(a^{2} / b^{2}\right)\right)^{2}} \\
+\frac{a^{4}}{8 b^{4}}+\frac{(a / b)^{4}}{\left(1+4\left(a^{2} / b^{2}\right)\right)^{2}}+\frac{(a / b)^{4}}{\left(4+\left(a^{2} / b^{2}\right)\right)^{2}}
\end{array}\right)
\end{array}\right)
\end{array}\right\}=\frac{16}{\pi^{2}}
$$




\section{Static Analysis}

As a further extension of the proposed SDOF approach, static loads were also taken into account. Besides, such a loading condition has no correlation with blast events, and the same equations ((25) and (26)) with slight modifications can be in fact efficiently used to predict the static response of a given elastic plate.

The governing equation is obtained from equation (25), by assuming $\ddot{Y}(t)=0$ and replacing $Y(t)$ with the static maximum deflection $Y_{\mathrm{m}}$. Therefore, original equations (25) and (26) take the following form:

$$
\begin{aligned}
w(x, y, t) & =Y_{m} \varphi(x, y, t), \\
K_{1}^{\text {st }} Y_{m}+K_{3}^{\text {st }} Y_{m}^{3} & =P_{0} .
\end{aligned}
$$

5.1. Simply Supported (SS) Edges. For general plates with all simply supported edges, the bending stiffness is defined as

$$
K_{1}=\frac{\pi^{4} D a b}{4} \sum_{m=1}^{\infty} \sum_{n=1}^{\infty}\left[\left(\frac{m}{a}\right)^{2}+\left(\frac{n}{b}\right)^{2}\right]^{2}
$$

while the membrane stiffness $K_{3}$ modifies with the edge condition.

For the immovably constrained edge condition, in particular, it is found that

$$
K_{3}=\frac{a b}{4}\left(\frac{\pi^{4} E h^{3}}{8 a^{4}}\right)\left[\begin{array}{c}
\frac{\sum_{n=1}^{\infty} n^{4} \beta^{4}+2 \nu \beta^{2} \sum_{m=1}^{\infty} \sum_{n=1}^{\infty} m^{2} n^{2}+\sum_{m=1}^{\infty} m^{4}}{1-\nu^{2}} \\
+\frac{1}{2}\left(1+\frac{a^{4}}{b^{4}}\right) \sum_{m=1}^{\infty} \sum_{n=1}^{\infty} m^{2} n^{2}
\end{array}\right] .
$$

For the stress-free edge condition:

$$
K_{3}=\frac{a b}{4}\left(\frac{2 \pi^{4} E h^{3}}{a^{2} b^{2}}\right)\left[\bar{\varphi}_{01}+\bar{\varphi}_{10}-\left(\frac{1}{32}\right)\left(\left(\frac{a^{2}}{b^{2}}\right)+\left(\frac{b^{2}}{a^{2}}\right)\right)\right] f^{3} .
$$

For the movable edge condition, it is

$$
K_{3}=\frac{a b}{4}\left(\frac{\pi^{4} E h^{3}}{16 a^{4}}\right)\left(1+\frac{a^{4}}{b^{4}}\right) \sum_{m=1}^{\infty} \sum_{n=1}^{\infty} m^{2} n^{2}
$$

where the force $P(t)$ is expressed as

$$
P(t)=\frac{4 a b}{(\pi)^{2}} \sum_{m=1,3,5, \ldots} \sum_{n=1,3,5, \ldots}\left(\frac{1}{m n}\right) P_{1}(t) .
$$

5.2. Clamped (CC) Edges. The bending stiffness $K_{1}$ is defined as

$$
K_{1}=\left(\frac{\pi^{4} D a b}{4}\right) \sum_{m=1}^{\infty} \sum_{n=1}^{\infty}\left[\left(\frac{3 m^{4}}{a^{4}}\right)+\left(\frac{2 m^{2} n^{2}}{a^{2} b^{2}}\right)+\left(\frac{3 n^{4}}{b^{4}}\right)\right] .
$$

Regarding the membrane stiffness $K_{3}$, for the immovably constrained edge condition, it is given by

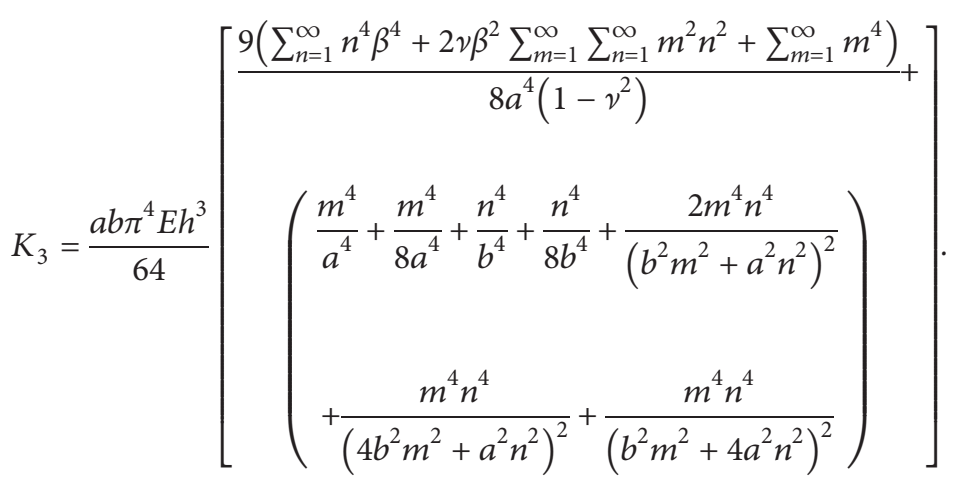


For the stress-free edge condition:

$$
K_{3}=\frac{a b \pi^{4} E h^{3}}{2 a^{2} b^{2}}\left(\begin{array}{c}
\bar{\varphi}_{01}+\bar{\varphi}_{10}+\bar{\varphi}_{11}+\frac{1}{2} \bar{\varphi}_{12}+\frac{1}{2} \bar{\varphi}_{21}+\bar{\varphi}_{02}+\bar{\varphi}_{20} \\
-\frac{1}{32}\left(\frac{a^{4}}{b^{4}}+\frac{9}{8}+\frac{2(a / b)^{4}}{\left(1+\left(a^{2} / b^{2}\right)\right)^{2}}+\frac{a^{4}}{8 b^{4}}+\frac{(a / b)^{4}}{\left(1+4\left(a^{2} / b^{2}\right)\right)^{2}}+\frac{(a / b)^{4}}{\left(4+\left(a^{2} / b^{2}\right)\right)^{2}}\right)
\end{array}\right) .
$$

For the movable edge conditions:

$$
K_{3}=\frac{a b \pi^{4} E h^{3}}{64 a^{2} b^{2}}\left(\left(\frac{a^{4}}{b^{4}}\right)+\left(\frac{9}{8}\right)+\frac{2(a / b)^{4}}{\left(1+\left(a^{2} / b^{2}\right)\right)^{2}}+\left(\frac{a^{4}}{8 b^{4}}\right)+\frac{(a / b)^{4}}{\left(1+4\left(a^{2} / b^{2}\right)\right)^{2}}+\frac{(a / b)^{4}}{\left(4+\left(a^{2} / b^{2}\right)\right)^{2}}\right) .
$$

The force $P(t)$ is given by

$$
P(t)=\left(\frac{a b}{4}\right)
$$

Again, considering equation (26) in a different form:

$$
\ddot{Y}(t)+\left(\frac{K_{1}}{m}\right) Y(t)+\left(\frac{K_{3}}{m}\right) Y^{3}(t)=\left(\frac{P(t)}{m}\right) .
$$

The latter can be further expressed in a normalized form:

$$
\ddot{Y}(t)+\omega^{2} Y(t)+\omega^{2} \in Y^{3}(t)=q_{0}(t) .
$$

The governing parameters calculated from the proposed SDOF model (equation (118)), for both SS and CC plates with various edge conditions, are finally reported in Table 1.

\section{Validation of the Enhanced SDOF Model}

For the validation of the presently developed SDOF model, the analytical results reported in [33] by Feldgun et al. for elastic thin plates were first taken into account. Figure 3, in this regard, shows the calculated stiffness coefficient $K_{1}$ for SS and CC plates, along with the past SDOF results from [33]. For both SS and CC plates and variable $a / b$ values, it can be observed that the results of the present SDOF approach are in close agreement with [33], and a mostly perfect match can be observed for various geometrical configurations.

Similarly, further validation was carried out for additional edge restraint conditions, namely, represented by immovable, movable, and stress-free edges (for both the SS and CC plates).

The corresponding results for the $K_{3}$ membrane stiffness coefficient are proposed in Figure 4. Also, in this case, a close match can be perceived from the present SDOF model and the past calculations presented in [33].

Finally, the current SDOF model estimates were also validated for additional combinations of edge conditions, as reported in Figure 5.

\section{Accuracy of SDOF Solutions for SS and CC Plates}

In order to check the accuracy of SDOF results, another derivation was carried out for SS and CC plates. In particular, it is shown that (for any value of $m$ and $n$ ) the results from the proposed SDOF approach converge and are exact. This is the intrinsic advantage of infinite trigonometric series function and therefore the major issue of sinusoidal loadbased SDOF formulations of literature.

To this aim, the deflection for SS or CC plates is first defined by equations (119) and (120), respectively.

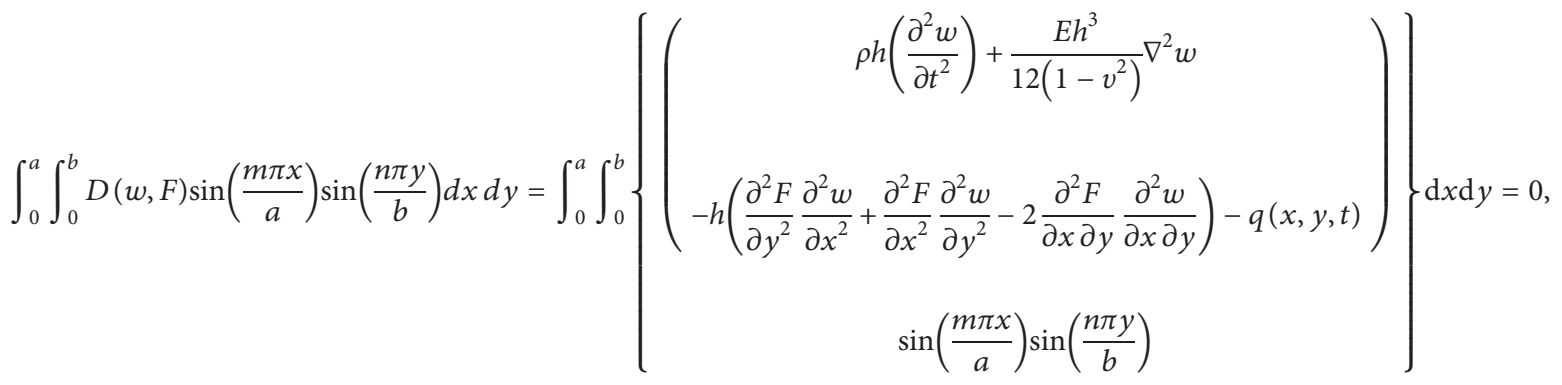


TABLE 1: Derivation of the governing parameters for SS or CC square plates, based on the SDOF model herein developed.

\begin{tabular}{|c|c|c|c|c|}
\hline Boundary condition & Edge condition & $q_{0}(t)$ & $\omega^{2}$ & $\epsilon$ \\
\hline \multirow{3}{*}{ Simply supported (SS) } & Immovably constrained & $\left(16 / \pi^{2} \rho \mathbf{h}^{2}\right) \mathbf{p}(\mathbf{t})$ & $\left(\pi^{4} \mathbf{E h}^{2} / 3 \rho\left(1-v^{2}\right) \mathbf{a}^{4}\right)$ & $\left(\begin{array}{c}(3 / 8)(3-v) \\
(1+v)\end{array}\right)$ \\
\hline & Stress-free & $\left(16 / \pi^{2} \rho \mathbf{h}^{2}\right) \mathbf{p}(\mathbf{t})$ & $\left(\pi^{4} \mathbf{E h}^{2} / 3 \rho\left(1-v^{2}\right) \mathbf{a}^{4}\right)$ & \multirow{2}{*}{$\begin{array}{l}\left(\begin{array}{c}0.19476 \\
\left(1-v^{2}\right)\end{array}\right) \\
(3 / 8)\left(1-v^{2}\right) \\
\end{array}$} \\
\hline & Movable edges & $\left(16 / \pi^{2} \rho \mathbf{h}^{2}\right) \mathbf{p}(\mathbf{t})$ & $\left(\pi^{4} \mathbf{E h}^{2} / 3 \rho\left(1-v^{2}\right) \mathbf{a}^{4}\right)$ & \\
\hline \multirow{3}{*}{ Clamped (CC) } & Immovably constrained & $\left(16 / \pi^{2} \rho \mathbf{h}^{2}\right) \mathbf{p}(\mathbf{t})$ & $\left(32 \pi^{4} \mathbf{E h}^{2} / 27 \rho\left(1-v^{2}\right) \mathbf{a}^{4}\right)$ & $\begin{array}{l}(27 / 128)(1+v)+ \\
(8.49 / 32)\left(1-v^{2}\right)\end{array}$ \\
\hline & Stress-free & $\left(16 / 9 \rho \mathbf{h}^{2}\right) \mathbf{p}(\mathbf{t})$ & $\left(32 \pi^{4} \mathbf{E h}^{2} / 27 \rho\left(1-v^{2}\right) \mathbf{a}^{4}\right)$ & \multirow{2}{*}{$\begin{array}{c}\left(\begin{array}{c}0.22355 \\
\left(\left(1-v^{2}\right)\right)\end{array}\right) \\
0.2653\left(1-v^{2}\right)\end{array}$} \\
\hline & Movable edges & $\left(16 / 9 \rho \mathbf{h}^{2}\right) \mathbf{p}(\mathbf{t})$ & $\left(32 \pi^{4} \mathbf{E h}^{2} / 27 \rho\left(1-v^{2}\right) \mathbf{a}^{4}\right)$ & \\
\hline
\end{tabular}

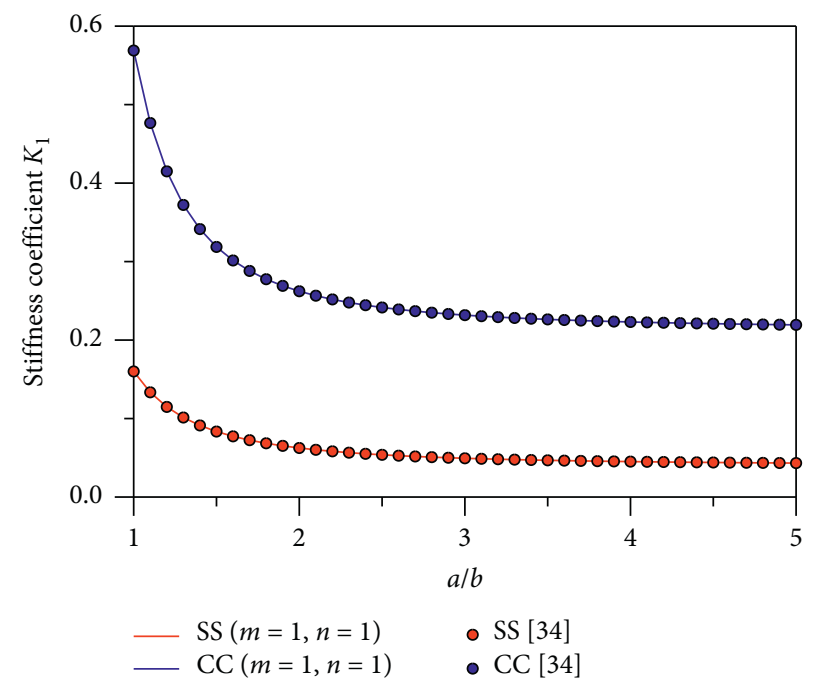

FIGURE 3: Comparison of bending stiffness predictions $\left(K_{1}\right)$, as obtained from the current SDOF model and literature [33].

$$
\int_{0}^{a} \int_{0}^{b} D(w, F) \sin ^{2}\left(\frac{m \pi x}{a}\right) \sin ^{2}\left(\frac{n \pi y}{b}\right) \mathrm{d} x \mathrm{~d} y=\int_{0}^{a} \int_{0}^{b}\left\{\left(\begin{array}{c}
\rho h \frac{\partial^{2} w}{\partial t^{2}}+\frac{E h^{3}}{12\left(1-v^{2}\right)} \nabla^{2} w \\
-h\left(\left(\frac{\partial^{2} F}{\partial y^{2}}\right)\left(\frac{\partial^{2} w}{\partial x^{2}}\right)+\left(\frac{\partial^{2} F}{\partial x^{2}}\right)\left(\frac{\partial^{2} w}{\partial y^{2}}\right)-2\left(\frac{\partial^{2} F}{\partial x \partial y}\right)\left(\frac{\partial^{2} w}{\partial x \partial y}\right)\right)-q(x, y, t) \\
\sin ^{2}\left(\frac{m \pi x}{a}\right) \sin ^{2}\left(\frac{n \pi y}{b}\right)
\end{array}\right\} \mathrm{d} x \mathrm{~d} y=0 .\right.
$$

The above equation can be rewritten in the following form:

$$
\int_{0}^{a} \int_{0}^{b} D(w, F) \sin \left(\frac{m \pi x}{a}\right) \sin \left(\frac{n \pi y}{b}\right) \mathrm{d} x \mathrm{~d} y=\int_{0}^{a} \int_{0}^{b}\left\{\left(-\left(N_{x x}\left(\frac{\partial^{2} w}{\partial y^{2}}\right)+\left(\frac{\partial^{2} w}{\partial x^{2}}\right) N_{y y}-2 N_{x y}\left(\frac{\partial^{2} w}{\partial x \partial y}\right)\right)-q(x, y, t)\right)\right\} \mathrm{d} x \mathrm{~d} y=0,
$$

where 


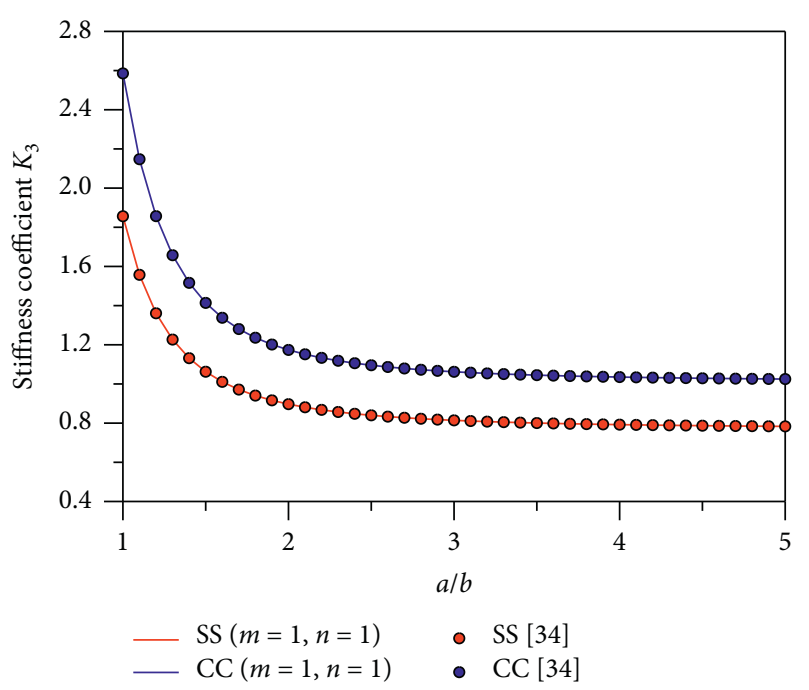

(a)

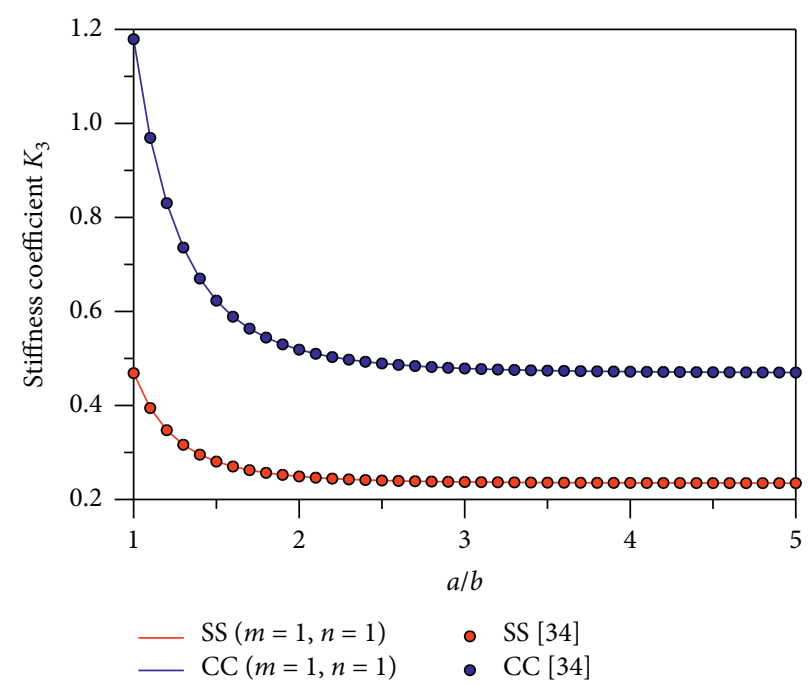

(b)

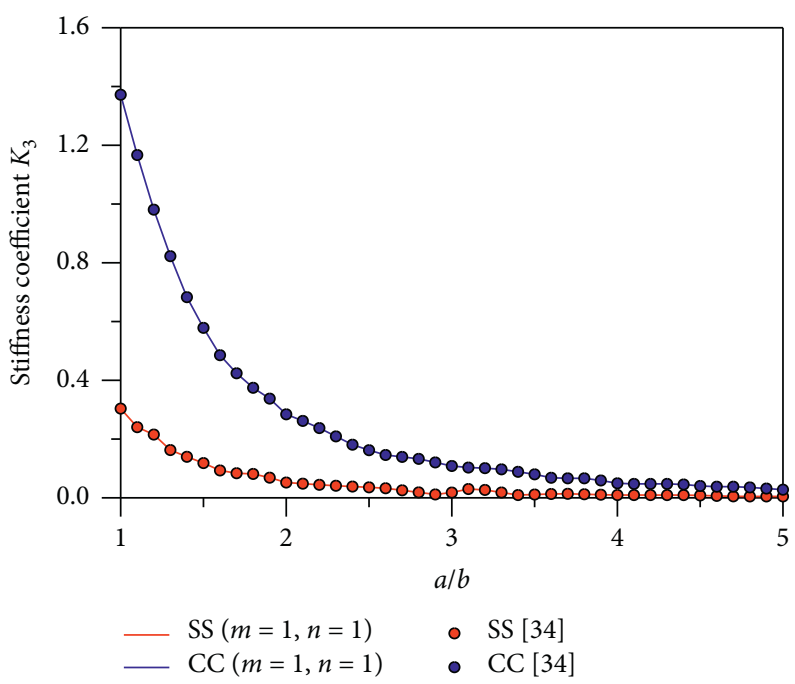

(c)

FIGURE 4: Comparison of membrane stiffness predictions $\left(K_{3}\right)$, as obtained from the current SDOF model and literature [33]. (a) Immovable edges. (b) Movable edges. (c) Stress-free edges.

$$
\begin{aligned}
& N_{x x}=h\left(\frac{\partial^{2} F}{\partial y^{2}}\right), \\
& N_{x y}=-h\left(\frac{\partial^{2} F}{\partial x \partial y}\right), \\
& N_{y y}=h\left(\frac{\partial^{2} F}{\partial x^{2}}\right),
\end{aligned}
$$

are the membrane forces. Using von Karman equations and focusing on the membrane and bending actions, for the SS plate, it is found that

$$
\begin{aligned}
& \left(\frac{m \pi}{a}\right)^{2}\left(N_{x x}\right)_{c r}+\left(\frac{n \pi}{b}\right)^{2}\left(N_{y y}\right)_{c r} \\
& =\frac{\pi^{4} D a b}{4}\left\{\sum_{m=1}^{\infty} \sum_{n=1}^{\infty}\left[\left(\frac{m}{a}\right)^{2}+\left(\frac{n}{b}\right)^{2}\right]^{2}\right\} \\
& \frac{m^{2}\left(N_{x x}\right)_{c r}}{\pi^{2} a^{2} D}+\frac{n^{2}\left(N_{y y}\right)_{c r}}{\pi^{2} b^{2} D}=\left(\frac{m^{2}}{a^{2}}+\frac{n^{2}}{b^{2}}\right)^{2} .
\end{aligned}
$$

Therefore, in order to predict the out-of-plane response of the plate, $m$ and $n$ are chosen in such a way that the values $N_{x x}$ and $N_{y y}$ are minimized for any given value of $N_{x x} / N_{y y}$. 


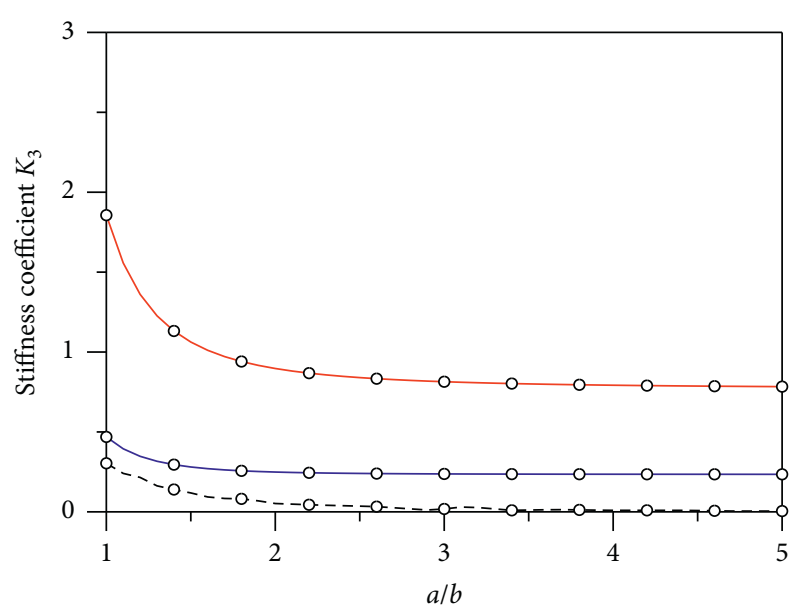

SS plate $(m=1, n=1)$

__ Immovable edges

- - Stress-free edges

- From [34]

(a)

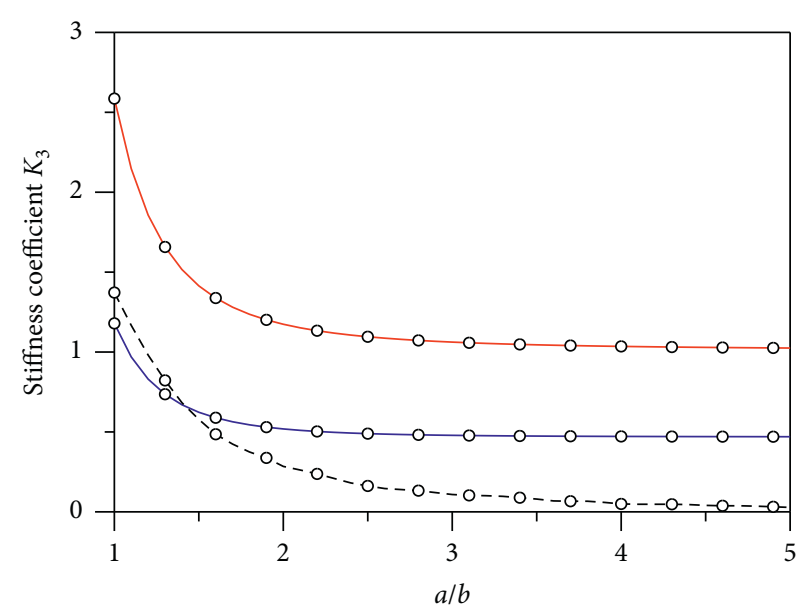

CC plate $(m=1, n=1)$

$\begin{array}{lcl}\text { _ Immovable edges } & -- & \text { Stress-free edges } \\ \text { _ Movable edges } & \circ & \text { From [34] }\end{array}$

(b)

FIgURE 5: Variation of the membrane stiffness coefficient $\left(K_{3}\right)$ as a function of $a / b$, as obtained with $m=1$ and $n=1$ for (a) SS and (b) CC plates with different edge conditions.

In the limit case of a square plate subjected to a uniform pressure $P(t)$ on its middle plane, it is $a=b$ and therefore $N_{x x}=N_{y y}$. Accordingly, equation (124) becomes representative of a critical value $\left(N_{x x}\right)_{c r}$ that is defined as

$$
\left(N_{x x}\right)_{c r}=\frac{\pi^{2} D}{a^{2}}\left(m^{2}+n^{2}\right)_{\min }
$$

For this limit condition only, the minimum $\left(N_{x x}\right)_{c r}$ value of equation (125) is achieved for $m=1$ and $n=1$. On the other hand, in the general case of rectangular plates, neither $N_{x x}$ nor $N_{y y}$ is able to converge at $m=1$ and $n=1$.

Hence,

$$
\begin{aligned}
F & =-\frac{E h^{2}}{32}\left(\frac{a^{2}}{b^{2}} \sum_{m=1}^{\infty} \cos \left(\frac{2 m \pi x}{a}\right)+\frac{b^{2}}{a^{2}} \sum_{n=1}^{\infty} \cos \left(\frac{2 n \pi y}{b}\right)\right), \\
N_{y y} & =h\left(\frac{\partial^{2} F}{\partial x^{2}}\right)=\frac{E h^{3}}{32}\left(\frac{2 m \pi}{a}\right)^{2} \frac{a^{2}}{b^{2}} \\
N_{x x} & =h\left(\frac{\partial^{2} F}{\partial y^{2}}\right)=\frac{E h^{3}}{32}\left(\frac{2 n \pi}{b}\right)^{2} \frac{b^{2}}{a^{2}} .
\end{aligned}
$$

The comparison of the above equations leads to

$$
\begin{aligned}
N_{y y} & =\frac{(2 m \pi / b)^{2}}{(2 n \pi / a)^{2}}, \\
N_{x x} & =\frac{m^{2}}{n^{2}} \frac{a^{2}}{b^{2}} N_{x x}, \\
\left(\frac{m^{2}\left(N_{x x}\right)_{c r}}{\pi^{2} a^{2} D}\right)+\left(\frac{n^{2}\left(N_{x x}\right)_{c r}}{\pi^{2} b^{2} D}\right)\left(\frac{m^{2}}{n^{2}}\right)\left(\frac{a^{2}}{b^{2}}\right) & =\left(\frac{m^{2}}{a^{2}}+\frac{n^{2}}{b^{2}}\right)^{2}, \\
\left(N_{x x}\right)_{c r} & \left.=\pi^{2} D\right)\left(m^{2} / a^{2}\right)+\left(\frac{\left.\left.n^{2} / b^{2}\right)\right)^{2}}{\left(\left(m^{2} / a^{2}\right)+\left(m^{2} a^{2} / b^{4}\right)\right) .}\right.
\end{aligned}
$$

Finally, given that the $\left(N_{x x}\right)_{c r}$ is obtained when $n=1$, it or follows that

$$
\left(N_{x x}\right)_{c r}=\pi^{2} D \frac{\left.\left(m^{2} / a^{2}\right)+\left(1 / b^{2}\right)\right)^{2}}{\left(\left(m^{2} / a^{2}\right)+\left(m^{2} a^{2} / b^{4}\right)\right)},
$$

$$
\left(N_{x x}\right)_{c r}=\pi^{2} D \frac{\left(m^{2}+\left(a^{2} / b^{2}\right)\right)^{2}}{\left(m^{2}+\left(m^{2} a^{4} / b^{4}\right)\right)},
$$


Accordingly, the smallest value of $N_{x x}$ at which the denominator of one of the terms in equations (128) and (129) becomes zero represents the critical value $\left(N_{x x}\right)_{c r}$.

Figure 6(a) shows the variation of $N_{x x}$ with the $a / b$ ratio for thin elastic plates. Comparative results are proposed for $m=1,2, \ldots, 6$. From the collected plots, it is possible to observe that all $N_{x x}$ are converging, but with the exception of the $m=1$ case. It must also be noted that the deflection expression for SS plates is an odd function. Consequently, it is necessarily required to choose the minimum converged odd $m$ value, to achieve the exact solution. For the calculation examples in Figure 6(a), the minimum critical value for SS plates is therefore $m=3$.

By following a similar procedure, the variation of $N_{x x}$ and $N_{y y}$ with $a / b$ can be found for CC plates. In the latter case, the maximum values of infinite trigonometric series function are represented by equations (130) and (131), respectively:

$$
N_{x x}=h \frac{\partial^{2} F}{\partial y^{2}}=-\left(\frac{1}{32}\right)\left[\begin{array}{c}
\frac{m^{2}}{n^{2} \beta^{2}}\left(\frac{2 n \pi}{b}\right)^{2}+\frac{m^{2} n^{2} \beta^{2}}{\left(4 m^{2}+n^{2} \beta^{2}\right)^{2}}\left(\frac{2 n \pi}{b}\right)^{2}+ \\
\frac{m^{2} n^{2} \beta^{2}}{\left(m^{2}+4 n^{2} \beta^{2}\right)^{2}}\left(\frac{4 n \pi}{b}\right)^{2} \\
-\frac{m^{2}}{16 n^{2} \beta^{2}}\left(\frac{4 n \pi}{b}\right)^{2}-\frac{m^{2} n^{2} \beta^{2}}{\left(m^{2}+n^{2} \beta^{2}\right)^{2}}\left(\frac{2 n \pi}{b}\right)^{2}
\end{array}\right],
$$

Therefore, by comparing each term in the above equations, it can be seen that they are defined by

$$
\begin{aligned}
N_{y y} & =\left(\frac{m^{2}}{n^{2}}\right)\left(\frac{a^{2}}{b^{2}}\right) N_{x x}, \\
\frac{m^{2}\left(N_{x x}\right)_{c r}}{\pi^{2} a^{2} D}+\frac{n^{2}\left(N_{x x}\right)_{c r}}{\pi^{2} b^{2} D}\left(\frac{m^{2}}{n^{2}}\right)\left(\frac{a^{2}}{b^{2}}\right) & =\frac{1}{2}\left(\left(\frac{3 m^{4}}{a^{4}}\right)+\left(\frac{2 m^{2} n^{2}}{a^{2} b^{2}}\right)+\left(\frac{3 n^{4}}{b^{4}}\right)\right), \\
\left(N_{x x}\right)_{c r} & =\frac{(1 / 2)\left(\left(\left(3 m^{4} / a^{4}\right)\right)+\left(2 m^{2} n^{2} / a^{2} b^{2}\right)+\left(3 n^{4} / b^{4}\right)\right)}{\left(m^{2} / \pi^{2} a^{2} D\right)+\left(n^{2} / \pi^{2} b^{2} D\right)\left(m^{2} / n^{2}\right)\left(a^{2} / b^{2}\right)} .
\end{aligned}
$$

The critical value $\left(N_{x x}\right)_{c r}$ is obtained by assuming $n=1$. Accordingly, the above equation becomes

$$
\left(N_{x x}\right)_{c r}=C_{1} \frac{\left(\left(3 m^{4} / a^{4}\right)+\left(2 m^{2} / a^{2} b^{2}\right)+\left(3 / b^{4}\right)\right)}{\left(m^{2} / a^{2}\right)+\left(m^{2} a^{2} / b^{2}\right),}
$$

or

$$
\left(N_{x x}\right)_{c r}=C_{1} \frac{\left(3 m^{4}+\left(2 a^{2} m^{2} / b^{2}\right)+\left(3 a^{4} / b^{4}\right)\right)}{m^{2}+\left(m^{2} a^{4} / b^{4}\right)},
$$

The so-calculated values are proposed in Figure 6(b).

As in the case of the SS plates, it can be still observed that the collected $N_{x x}$ values are not converging for $m=1$, but they are indeed converging for all other cases $(m=2,3,4,5$, 

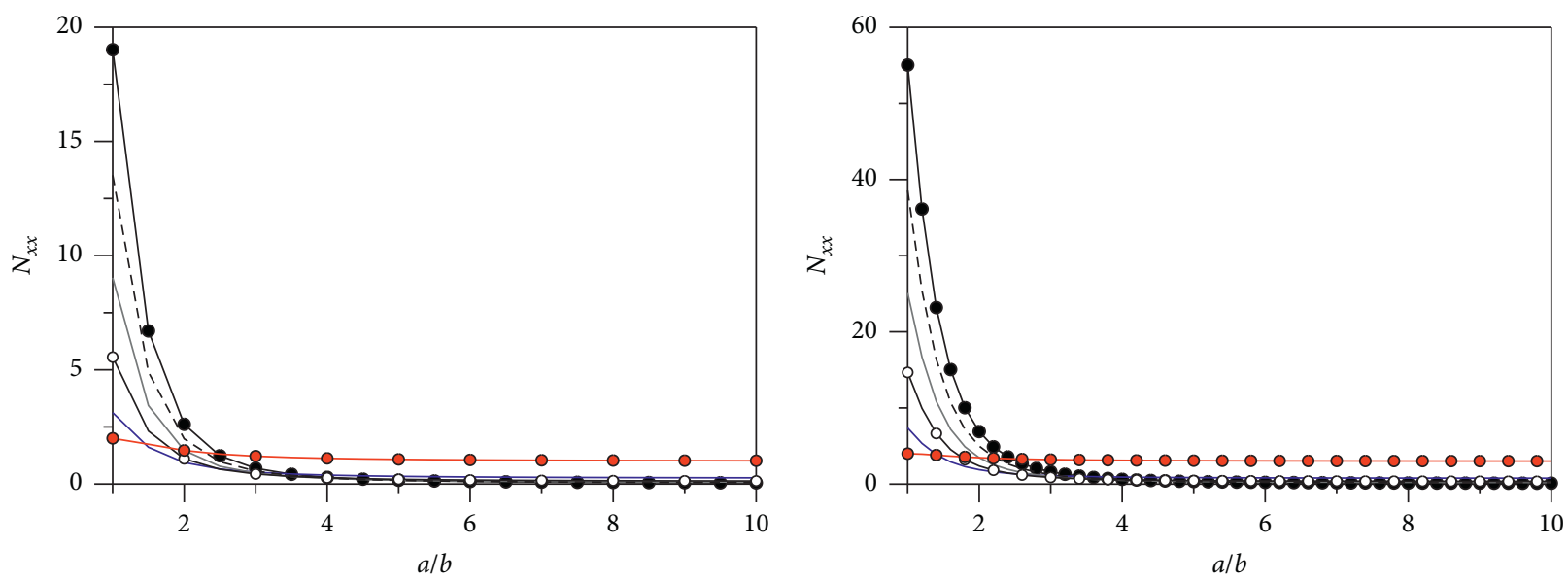

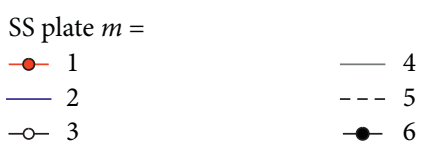

(a)

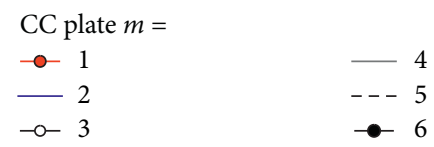

(b)

Figure 6: Variation of $N_{x x}$ as a function of $a / b$ : (a) SS plates and (b) CC plates.

6). The exact solution for CC plates is given by the minimum converged value, that is, $m=2$ from Figure 6(b).

\section{Experimental Validation for Square Plates under Blast}

In order to further assess the reliability of SDOF estimates, additional comparative calculations were carried out by taking into account the experimental study reported in [38] for ship panels subjected to air blast waves.

In [38], in particular, Houlston et al. carried out a series of field experiments (12 repetitions in total) on square steel plates with an effective span of $508 \mathrm{~mm}$ and a thickness of $3.4 \mathrm{~mm}$. Young's modulus of elasticity was $E=207 \mathrm{GPa}$, with $\nu=0.3$ being Poisson's ratio and $\rho=7770 \mathrm{~kg} / \mathrm{m}^{3}$ being the mass density. No dynamic increase factor (DIF) has been taken into account in [38], for the reference material properties. In this paper, for comparative and validation purposes, the same input parameters were taken into account, and any DIF was still disregarded.

The dynamic response of the reference steel plate has been explored in [38] under a typical blast pressure-time history agreeing with Figure 7 . The measured pressure from the experimental trial (three transducers) is reported, together with an average fitting pressure history. The typical test setup has been thus arranged so as to include the steel target plate, with fully clamped (CC) edges and subjected to uniform pressure. The restraint details have been realized by means of four bolted steel box beams, clamped to a concrete base seated on the ground.

As far as the fitting pressure history of Figure 7 is taken into account and uniformly distributed on the plate surface for the SDOF model herein proposed (with both the positive and negative phases included), major calculation results are collected in Figure 8, where the out-of-plane displacement at the center of the plate is shown. More in detail, both the

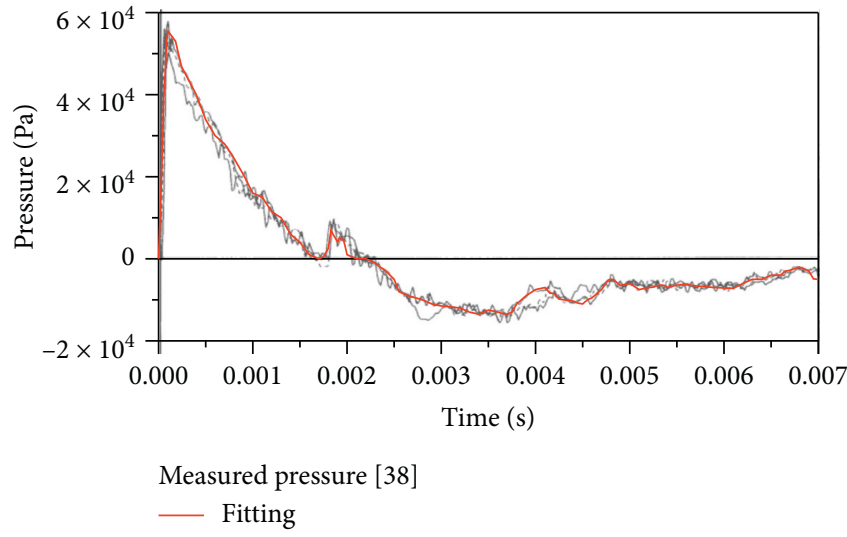

FIgURE 7: Reference field blast pressure time-history (adapted from [38] with permission from Elsevier ${ }^{\circledR}$, Copyright license number: 4853550044917, June 2020).

linear and nonlinear approaches are compared for the CC boundary configuration. Together with the past experimental estimates from [38], additional comparative data are proposed for the same steel plate, as obtained from FE nonlinear dynamic analysis reported in [38], as well as from the SDOF formulation presented in [33].

In Figure 8, it can be observed that the presently developed SDOF model is in close agreement with the displacement predictions from earlier researchers. Further, for both the linear and nonlinear CC plates, the current SDOF model estimates are in rather good agreement with the SDOF results given in [33]. As such, it can be concluded that the present SDOF model predicts the measured time history very well and provides good estimates both for displacement amplitudes and periods of vibration.

As far as the SS condition is also assessed for the same steel plates, as also expected, Figure 9 shows an increased 

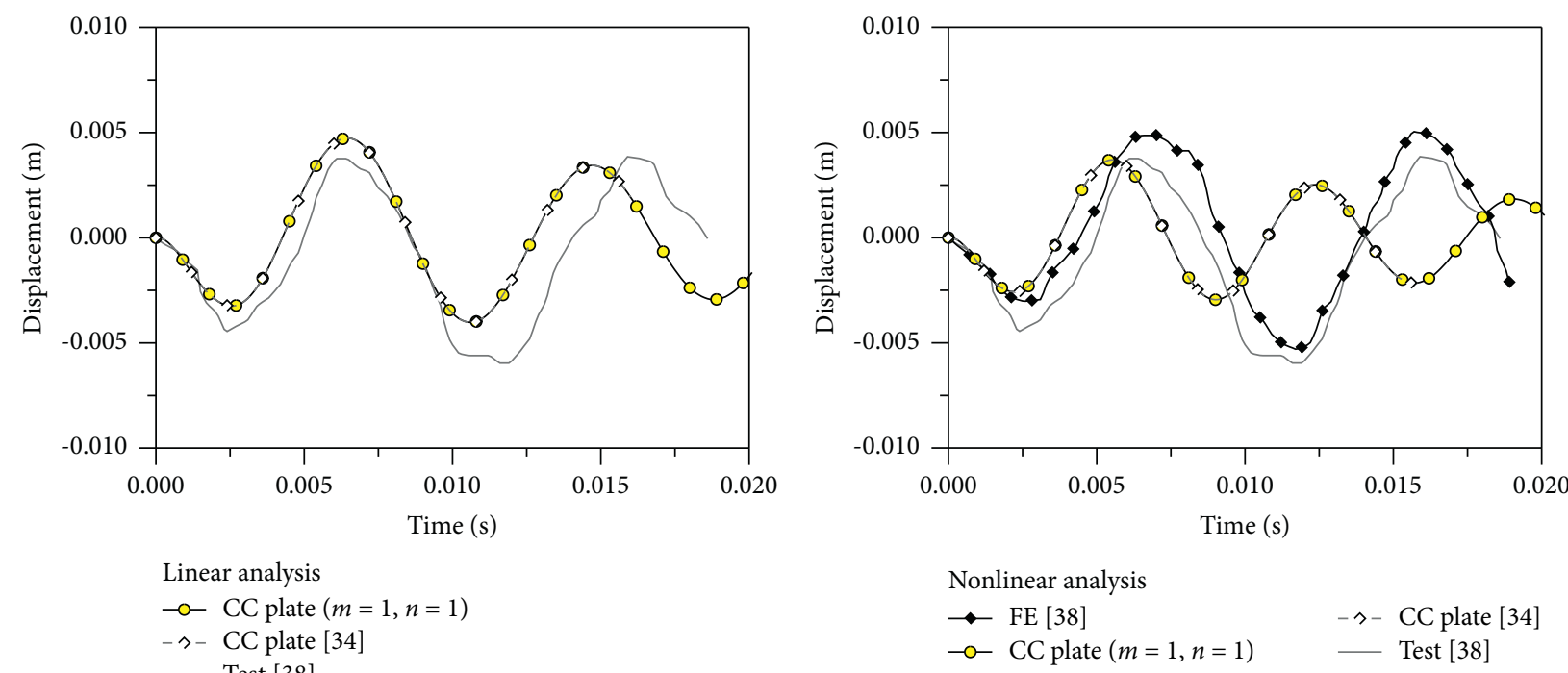

(a)

(b)

FIGURE 8: Out-of-plane displacement for a square steel plate under field blast loads, as obtained from the presently developed SDOF model and from literature $[33,38]$. In evidence, the results are given by the (a) linear and (b) nonlinear solutions for the CC boundary condition.

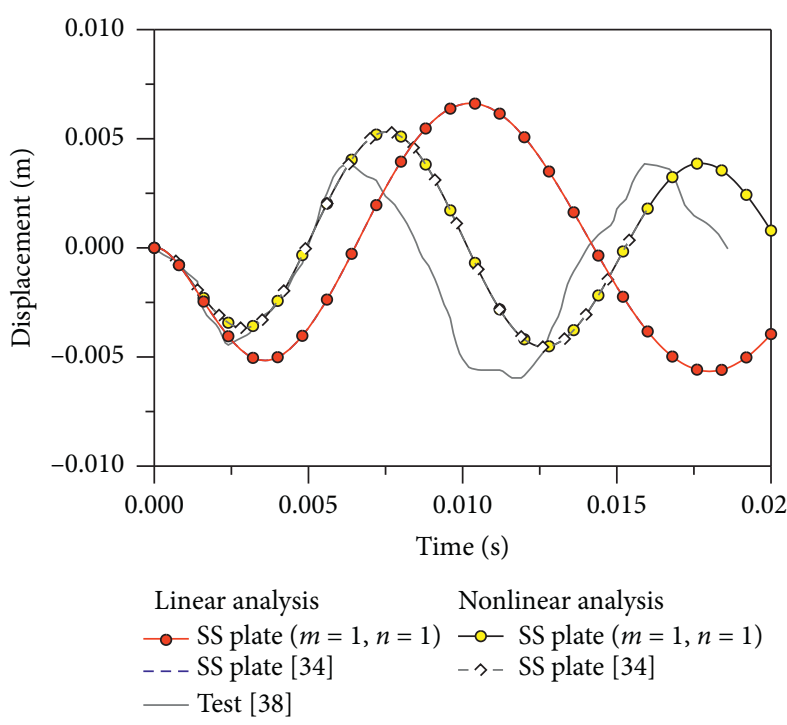

FIGURE 9: Out-of-plane displacement for a square steel plate under field blast loads, as obtained from the presently developed SDOF model and from literature $[33,38]$. In evidence, the results are given by the linear and nonlinear solutions for the SS boundary condition.

scatter of the SDOF calculations from the corresponding experimental estimates. As such, this further emphasizes the need of analytical solutions that could be adapted to multiple restraint conditions of technical interest. On the other hand, the agreement of the displacement plots with the past SDOF predictions from [33] is still good.

\section{Conclusions}

Single-degree-of-freedom (SDOF) methods can offer a robust support in the analysis of structural systems. On the other hand, the basic assumptions can often involve approximations and misleading estimates. This can be the case of thin elastic plates under blast loads, where special care among other aspects should be given for the load description.

In this paper, an enhanced SDOF formulation was developed for the linear and nonlinear analysis of thin elastic plates under blast waves. The key governing parameters have been proposed, more in detail, for different boundary conditions of technical interest. Based on an energy approach, both the linear and nonlinear solutions were explored, by accounting, respectively, for the bending stiffness only or for both the bending and membrane stiffness contributions.

Various parameters (such as deflections, membrane stresses, and bending stresses) have been assessed for blastloaded plates with simply supported or clamped restraints, under different edge conditions (including movable, immovable, or membrane stress-free cases), under the effects of a uniform blast pressure.

Compared to existing SDOF formulation, as shown, the major advantage was represented by the use of infinite trigonometric series for the description of incoming blast pressures. The developed SDOF model has been therefore compared, for validation purposes, in terms of predicted stiffness coefficients as well as out-of-plane deflections in time, for a given blast wave. In doing so, major advantage was taken from available experimental and numerical solutions of literature. For both static and dynamic nonlinear solutions reported in the paper, the validity and accuracy of the proposed SDOF model have therefore been discussed.

\section{Data Availability}

The research data will be available upon request. 


\section{Conflicts of Interest}

The authors declare that there are no conflicts of interest regarding the publication of this paper.

\section{Acknowledgments}

The lead author, on behalf of all the authors, acknowledges the Ministry of Human Resources and Development (MHRD), Govt. of India, for the funding received for this research study (fellowship grants to the involved students, for completing the investigation herein reported). The lead author is also thankful to the SERB (DST), Govt. of India, for the funding received for this research study under ECRA scheme (ECR/2017/000941).

\section{References}

[1] G. Chandrasekharappa and H. R. Srirangarajan, "Nonlinear response of elastic plates to pulse excitations," Computers \& Structures, vol. 27, no. 3, pp. 373-378, 1987.

[2] X. Zhang and H. Hao, "The response of glass window systems to blast loadings: an overview," International Journal of Protective Structures, vol. 7, no. 1, pp. 123-154, 2016.

[3] P. W. Sielicki, C. Bedon, and X. Zhang, "Performance of TGU windows under explosive loading," in Soft Target Protection. NATO Science for Peace and Security Series C: Environmental Security, L. Hofreiter, V. Berezutskyi, L. Figuli, and Z. Zvaková, Eds., Springer, Dordrecht, Netherlands, 2020.

[4] L. Figuli, Z. Zvaková, and C. Bedon, "Design and analysis of blast loaded windows," Procedia Engineering, vol. 192, pp. 177-182, 2017.

[5] R. Sriram, U. Vaidya, and J.-E. Kim, "Blast impact response of aluminum foam sandwich composites," Journal of Materials Science, vol. 41, pp. 4023-4039, 2006.

[6] G. S. Langdon, C. J. von Klemperer, B. K. Rowland, and G. N. Nurick, "The response of sandwich structures with composite face sheets and polymer foam cores to air-blast loading: preliminary experiments," Engineering Structures, vol. 36, pp. 104-112, 2012.

[7] B. Schimizze, S. F. Son, R. Goel, A. P. Vechart, and L. Young, "An experimental and numerical study of blast induced shock wave mitigation in sandwich structures," Applied Acoustics, vol. 74, no. 1, pp. 1-9, 2013.

[8] M. D. Goel, V. A. Matsagar, S. Marburg, and A. K. Gupta, "Comparative performance of stiffened sandwich foam panels under impulsive loading," Journal of Performance of Constructed Facilities, vol. 27, no. 5, pp. 540-549, 2013.

[9] M. Yazici, J. Wright, D. Bertin, and A. Shukla, "Experimental and numerical study of foam filled corrugated core steel sandwich structures subjected to blast loading," Composite Structures, vol. 110, pp. 98-109, 2014.

[10] M. D. Goel and V. A. Matsagar, "Blast-resistant design of structures," Practice Periodical on Structural Design and Construction (ASCE), vol. 19, pp. 1-9, 2014.

[11] M. D. Goel, V. A. Matsagar, and A. K. Gupta, "Blast resistance of stiffened sandwich panels with closed-cell aluminum foam," Latin American Journal of Solids and Structures, vol. 11, no. 13, pp. 2497-2515, 2014.

[12] M. D. Goel, V. A. Matsagar, and A. K. Gupta, "Blast resistance of stiffened sandwich panels with aluminum cenosphere syntactic foam," International Journal of Impact Engineering, vol. 77, pp. 134-146, 2015.
[13] X. Q. Zhao and H. Hao, "Prediction of airblast loads on structures behind a protective barrier," International Journal of Impact Engineering, vol. 35, no. 5, pp. 363-375, 2008.

[14] P. D. Smith, "Blast walls for structural protection against high explosive threats: a Review," International Journal of Protective Structures, vol. 1, no. 1, pp. 67-84, 2009.

[15] W. Chen and H. Hao, "Numerical study of new multi arch double layered blast resistant door panel," International Journal of Impact Engineering, vol. 43, pp. 16-28, 2011.

[16] N. Aitavade, N. D. Jadhav, R. D. Thorat, and R. S. Ramavat, "Design and analysis of single plate blast resistant door," International Journal on Theoretical and Applied Research in Mechanical Engineering, vol. 2, pp. 107-111, 2013.

[17] S. Palla and M. D. Goel, "Performance assessment of curved steel wall under blast loading," International Journal of Science, Engineering and Management, vol. 2, no. 3, pp. 406-411, 2017.

[18] C. Bedon and L. Kruszka, "Failure simulation of brick protective walls under explosive aerial shock waves," in Critical Infrastructure Protection, et al. IOS Press, Amsterdam, Netherlands, 2019.

[19] M. Larcher, M. Arrigoni, C. Bedon et al., "Design of blastloaded glazing windows and facades: a review of essential requirements towards standardization," Advances in Civil Engineering, vol. 2016, p. 14, Article ID 2604232, 2016.

[20] H. Chu and G. Herrmann, "Influence of large amplitudes of free flexural vibrations of rectangular elastic plates," Technical report 532-540, Columbia University Department of Civil Engineering and Engineering Mechanics, New York, NY, USA, 1956.

[21] N. Yamaki, "Influence of large amplitudes on flexural vibrations of elastic plates," ZAMM - Zeitschrift für Angewandte Mathematik und Mechanik, vol. 41, no. 12, pp. 501-510, 1961.

[22] J. G. Eisley, "Nonlinear vibration of beams and rectangular plates," Zeitschrift für angewandte Mathematik und Physik ZAMP, vol. 15, no. 2, pp. 167-175, 1964.

[23] A. L. Florence, "Clamped circular rigid-plastic plates under central blast loading," International Journal of Solids and Structures, vol. 2, no. 2, pp. 319-335, 1966.

[24] K. T. S. R. Iyengar and M. M. Naqvi, "Large deflections of rectangular plates," International Journal of Non-linear Mechanics, vol. 1, no. 2, pp. 109-122, 1966.

[25] H. F. Bauer, "Nonlinear response of elastic plates to pulse excitations," Journal of Applied Mechanics, vol. 35, no. 1, pp. 47-52, 1968.

[26] D. J. Bayles, R. L. Lowery, and D. E. Boyd, "A non-linear dynamic lumped-parameter model of a rectangular plate," Journal of Sound and Vibration, vol. 21, no. 3, pp. 329-337, 1972.

[27] H. R. Srirangarajan and P. Srinivasan, "The pulse response of non-linear systems," Journal of Sound and Vibration, vol. 44, no. 3, pp. 369-377, 1976.

[28] C. M. Wang, G. T. Lim, J. N. Reddy, and K. H. Lee, "Relationships between bending solutions of Reissner and Mindlin plate theories," Engineering Structures, vol. 23, no. 7, pp. 838-849, 2001.

[29] M. Li and Q. H. Meng, "Pressure-impulse diagram for blast loads based on dimensional analysis and single-degree-offreedom model," Journal of Engineering Mechanics, vol. 128, pp. 87-92, 2002.

[30] K. Fischer and I. Häring, "SDOF response model parameters from dynamic blast loading experiments," Engineering Structures, vol. 31, no. 8, pp. 1677-1686, 2009. 
[31] W. El-Dakhakhni, W. F. Mekkay, and S. H. Changiz Rezaei, "Validity of SDOF models for analyzing two-way reinforced concrete panels under blast loading," Journal of Performance of Constructed Facilities, vol. 24, pp. 311-325, 2010.

[32] S. E. Rigby, A. Tyas, and T. Bennett, "Single-Degree-ofFreedom response of finite targets subjected to blast loading-the influence of clearing," Engineering Structures, vol. 45, pp. 396-404, 2012.

[33] V. R. Feldgun, D. Z. Yankelevsky, and Y. S. Karinski, "A nonlinear SDOF model for blast response simulation of elastic thin rectangular plates," International Journal of Impact Engineering, vol. 88, pp. 172-188, 2016.

[34] T. Mirzababaie Mostofi, H. Babaei, and M. Alitavoli, "Theoretical analysis on the effect of uniform and localized impulsive loading on the dynamic plastic behaviour of fully clamped thin quadrangular plates," Thin-Walled Structures, vol. 109, pp. 367-376, 2016.

[35] Q. M. Li and N. Jones, "Blast loading of fully clamped circular plates with transverse shear effects," International Journal of Solids and Structures, vol. 31, no. 14, pp. 1861-1876, 1994.

[36] J. Yan, Y. Liu, and F. Huang, "Improved SDOF approach to incorporate the effects of axial loads on the dynamic responses of steel columns subjected to blast loads," Advances in Civil Engineering, vol. 2019, Article ID 7810542, 9 pages, 2019.

[37] UFC 3-340-02, Structures to Resist the Effects of Accidental Explosions, Unified Facilities Criteria Program, U.S. Department of Defense, Arlington, VA, USA, 2008.

[38] R. Houlston, J. E. Slater, N. Pegg, and C. G. desrochers, “On analysis of structural response of ship panels subjected to air blast loading," Computers \& Structures, vol. 21, no. 1-2, pp. 273-289, 1985. 